UNIVERSITÀ DEGLI STUDI DI BERGAMO

DIPARTIMENTO DI INGEGNERIA DELL'INFORMAZIONE

E METODI MATEMATICI ${ }^{\circ}$

QUADERNI DEL DIPARTIMENTO

Department of Information Technology and Mathematical Methods

Working Paper

Series "Mathematics and Statistics"

n. $10 / \mathrm{MS}-2009$

Analysis and optimization of Robin-Robin partitioned

procedures in fluid-structure interaction problems

by

L. Gerardo-Giorda, F. Nobile, C. Vergara 
COMITATO DI REDAZIONE ${ }^{\S}$

Series Information Technology (IT): Stefano Paraboschi

Series Mathematics and Statistics (MS): Luca Brandolini, Ilia Negri

\footnotetext{
$\S$ L'accesso alle Series è approvato dal Comitato di Redazione. I Working Papers della Collana dei Quaderni del Dipartimento di Ingegneria dell'Informazione e Metodi Matematici costituiscono un servizio atto a fornire la tempestiva divulgazione dei risultati dell'attività di ricerca, siano essi in forma provvisoria o definitiva.
} 


\title{
ANALYSIS AND OPTIMIZATION OF ROBIN-ROBIN PARTITIONED PROCEDURES IN FLUID-STRUCTURE INTERACTION PROBLEMS *
}

\author{
LUCA GERARDO-GIORDA ${ }^{\dagger}$, FABIO NOBILE ${ }^{\ddagger}$, AND CHRISTIAN VERGARA
}

\begin{abstract}
In the solution of Fluid-Structure Interaction problems, partitioned procedures are modular algorithms that involve separate fluid and structure solvers, that interact, in an iterative framework, through the exchange of suitable transmission conditions at the FS interface. In this work we study, using Fourier analysis, the convergence of partitioned algorithms based on Robin transmission conditions. We derive, for different models of the fluid and the structure, a frequency dependent reduction factor at each iteration of the partitioned algorithm, which is minimized by choosing optimal values of the coefficients in the Robin transmission conditions. Two-dimensional numerical results are also reported, which highlight the effectiveness of the optimization procedure.
\end{abstract}

Key words. Fluid-structure interaction, Robin conditions, Fourier analysis, optimized Schwartz methods

AMS subject classifications. 65B99, 65M60,

1. Introduction. In the framework of Domain-Decomposition methods for the solution of differential problems, one of the most popular non-overlapping method is the one based on successive exchanges of interface Robin data and called Lions' method (see [18] and, e.g., [7, 16]). Recently, this strategy has been applied to the Fluid-Structure Interation (FSI) problem, which describes any physical phenomenon where a fluid and a structure interact by exchanging normal stresses through an interface (see [3]).

The solution of the FSI problem is problematic, since the fluid and the structure subproblems are coupled through the geometry problem (that is the determination of the unknown interface position) and through the interface continuity conditions, namely the continuity of the velocity and of the normal stresses at the FS interface.

Whatever treatment for the geometry problem is considered (e.g. fixed point, Newton, explicit extrapolation in time), a sequence of linearized FSI problems, implicitely coupled through the interface conditions, has to be solved (see Sect. 2.1). Implicit coupling of the interface conditions is required in many applications featuring a large added mass effect of the fluid on the structure (see [6]) and it allows to achieve at the numerical level perfect energy balance between fluid and structure.

One possible strategy for the solution of such problems is to consider modular algorithms (also referred to as partitioned procedures), that involve separate fluid and structure solvers in an iterative framework. In particular, they interact through the exchange of suitable transmission conditions at the FS interface, and guarantee, at convergence, the satisfaction of the continuity conditions. Modular algorithms can be reinterpreted, in the framework of the Domain-Decomposition method, as preconditioned Richardson iterations over a suitable interface equation (see $[2,8]$ ).

*The research of the second and of the third author has been (partially) supported by the ERC Advanced Grant N.227058 MATHCARD.

$\dagger$ Department of Mathematics and Computer Science, Emory University, Atlanta, GA, USA (luca@mathcs.emory.edu).

${ }^{\ddagger}$ MOX - Department of Mathematics - Politecnico di Milano, Milan, Italy (fabio.nobile@polimi.it).

$\S$ Department of Information Engineering and Mathematical Methods, Università degli Studi di Bergamo, Bergamo, Italy (chrstian.vergara@unibg.it). 
This allows one to introduce more performing Krylov methods for the solution of the FSI problem. In particular we mention the Dirichlet-Neumann/GMRES and the Robin-Neumann/GMRES schemes, which lead to different modular algorithms $($ see $[2,4])$.

The introduction of Robin-Robin (RR) partitioned procedures in the framework of FSI problems, as generalization of the classic Dirichelt-Neumann (DN) scheme, has been motivated to overcome the limitations of the latter algorithm. In particular, the performances of the DN scheme when the added mass effect is high (that is when the fluid and structure densities are similar) are very poor, and a (sometimes big) relaxation is needed to reach convergence (see $[6,12,23])$. On the contrary, RR schemes highlighted better convergence properties in the presence of a high addedmass. In particular, in $[2,3]$ the Robin-Neumann (RN) scheme has been shown to converge without relaxation, in the test cases studied, and to feature a big saving in computational time with respect to DN scheme.

This behaviour has been mostly evidenced by numerical tests and only few theoretical results of the convergence properties of partitioned procedures for the FSI problems are available so far. In particular, at the best of the authors' knowledge, the only convergence analysis have been proposed in [6] for the DN scheme and in [3] for the RN scheme. We mention also the analysis in [4] and in [2] for DN-GMRES and RN-GMRES schemes. In all the cases, the analysis has been performed on a simplified problem where the fluid is described by a $2 \mathrm{D}$ potential flow and the structure by a $1 \mathrm{D}$ reduced model (the independent rings in [6] and the generalized string in the other works).

The first goal of this work is to extend the convergence analysis of RR schemes (and then of DN) to more general classes of subproblems. In particular, we consider the generalized Stokes equations to describe the discretized-in-time fluid problem and a 2D linear elastic incompressible structure in the half plane. In particular, the proposed analysis are based on the application of the Fourier transform (see, e.g, $[1,13,14])$ and on the determination of a reduction factor.

Secondly, we focus on parameters in Robin transmission conditions. Obviously, the convergence velocity of RR schemes heavily depends on the choice of these parameters. A proposal for these parameters has been given in [3], based on heuristic considerations. This choice has revealed to be satisfactory in order to overcome the limitations of the DN scheme, but a rigorous optimization procedure is still missing.

The second goal of this work is to optimize the values of parameters in the Robin interface conditions, starting from the new convergence analysis proposed in this work and following the literature on Optimization Schwartz Methods (see $[1,13,14]$ for instance).

The outline of the paper is as follows. In Sect. 2 we introduce the FSI problem under investigation, we describe the different treatments of the geometry problem and we introduce the RR partitioned procedure. In Sect. 3 we perform the new convergence analysis, extending the results obtained in $[3,6]$ to more general classes of FSI problems. In Sect. 4 we propose an optimization procedure for the values of the parameters in Robin transmission conditions, starting from the convergence analysis. Finally, in Sect. 5 we present 2D numerical results, which confirm the effectiveness of the proposed optimal values with respect to the heuristic ones.

2. Problem setting. Let us consider a computational domain $\Omega^{t} \subset \mathbb{R}^{d}(d=2,3$, being the space dimension). This domain is divided into a sub-domain $\Omega_{s}^{t}$ occupied by an elastic structure and its complement $\Omega_{f}^{t}$ occupied by the fluid. The fluid-structure 
interface $\Sigma^{t}$ is the common boundary between $\Omega_{s}^{t}$ and $\Omega_{f}^{t}$ (see Fig. 2.1), while with $\Gamma_{i}^{t}$ and $\Gamma_{i, s}^{t}$ we denote the fluid and structure artificial sections. Furthermore, $\boldsymbol{n}$ is the outward normal on $\partial \Omega_{f}^{t}$. The initial configuration $\Omega^{0}$ at $t=0$ is considered as the reference one.
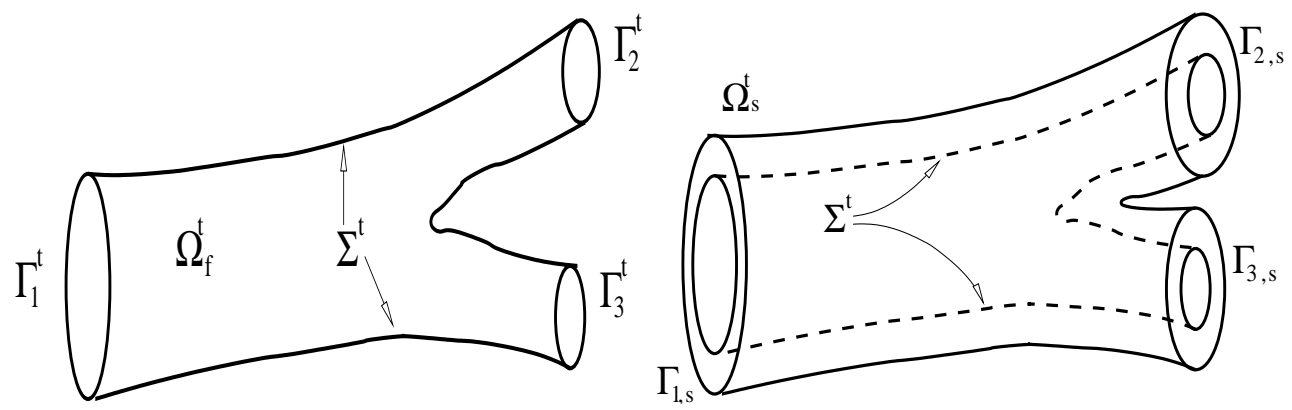

FIG. 2.1. Example of computational fluid domain $\Omega_{f}^{t}$ (left) and solid domain $\Omega_{s}^{t}$ (right).

We adopt a purely Lagrangian approach to describe the structure kinematics. We denote the reference (initial) configuration by $\Omega_{s}:=\Omega_{s}^{0}$. Hereafter, $\overline{\boldsymbol{\eta}}$ denotes the displacement of the solid medium with respect to $\Omega_{s}$. For any function $\bar{g}$ defined in the reference solid configuration, we denote by $g$ its counterpart in the current configuration. The solid is assumed to be a linear elastic material, characterized by the Cauchy stress tensor

$$
\boldsymbol{T}_{s}(\boldsymbol{\eta})=\lambda_{1}\left(\nabla \boldsymbol{\eta}+(\nabla \boldsymbol{\eta})^{T}\right)+\lambda_{2}(\nabla \cdot \boldsymbol{\eta}) \boldsymbol{I}
$$

where

$$
\lambda_{1}=\frac{E}{1+\nu}, \quad \lambda_{2}=\frac{E \nu}{(1+\nu)(1-2 \nu)}+\lambda_{1}
$$

are the Lamé constants, $E$ is the Young modulus, $\nu$ the Poisson modulus and $\boldsymbol{I}$ is the identity tensor.

On the other hand, the fluid problem is stated in an Arbitrary LagrangianEulerian (ALE) framework (see, e.g., $[9,17])$. The ALE mapping is defined by an appropriate lifting of the structure displacement. A classical choice is to consider a harmonic extension operator in the reference domain. In order to write the fluid problem in ALE coordinates, we recall the definition of ALE time derivative of the velocity $\boldsymbol{u}$ :

$$
\frac{D^{A} \boldsymbol{u}}{D t}=\frac{\partial \boldsymbol{u}}{\partial t}+\boldsymbol{w} \cdot \nabla \boldsymbol{u}
$$

where $\partial \boldsymbol{u} / \partial t$ is the Eulerian derivative and $\boldsymbol{w}$ is the velocity of the points of the fluid domain defined by the ALE map. Moreover, the fluid is assumed to be homogeneous, Newtonian and incompressible, with Cauchy stress tensor given by

$$
\boldsymbol{T}_{f}(\boldsymbol{u}, p)=-p \boldsymbol{I}+\mu\left(\nabla \boldsymbol{u}+(\nabla \boldsymbol{u})^{T}\right),
$$

where $p$ is the pressure and $\mu$ the dynamic viscosity.

Then, the full FSI problem in strong form reads: 
1. Fluid-structure problem. Find the fluid velocity $\boldsymbol{u}$, pressure $p$ and the structure displacement $\overline{\boldsymbol{\eta}}$ such that

$$
\begin{cases}\rho_{f} \frac{D^{A} \boldsymbol{u}}{D t}+\rho_{f}((\boldsymbol{u}-\boldsymbol{w}) \cdot \nabla) \boldsymbol{u}-\nabla \cdot \boldsymbol{T}_{f}=\boldsymbol{f}_{f} & \text { in } \Omega_{f}^{t} \times(0, T), \\ \nabla \cdot \boldsymbol{u}=0 & \text { in } \Omega_{f}^{t} \times(0, T), \\ \rho_{s} \frac{\partial^{2} \overline{\boldsymbol{\eta}}}{\partial t^{2}}-\bar{\nabla} \cdot \overline{\boldsymbol{T}}_{s}=\overline{\boldsymbol{f}}_{s} & \text { in } \Omega_{s}^{0} \times(0, T), \\ \boldsymbol{u}=\frac{\partial \boldsymbol{\eta}}{\partial t} & \text { on } \Sigma^{t} \times(0, T), \\ \boldsymbol{T}_{f} \boldsymbol{n}-\boldsymbol{T}_{s} \boldsymbol{n}=\mathbf{0} & \text { on } \Sigma^{t} \times(0, T),\end{cases}
$$

2. Geometry problem. Given the interface structure displacement $\left.\boldsymbol{\eta}\right|_{\Sigma^{t}}$, find a map $\mathcal{A}: \Omega_{f}^{0} \rightarrow \Omega_{f}^{t}$ e.g. through an harmonic extension Ext of the boundary displacement

$$
\mathcal{A}^{t}\left(\boldsymbol{x}_{0}\right)=\boldsymbol{x}_{0}+\operatorname{Ext}\left(\left.\overline{\boldsymbol{\eta}}\right|_{\Sigma^{0}}\right),
$$

such that $\Omega_{f}^{t}=\mathcal{A}^{t}\left(\Omega_{f}^{0}\right)$.

Here, $\rho_{f}$ and $\rho_{s}$ are the fluid and structure density and $\boldsymbol{f}_{f}$ and $\overline{\boldsymbol{f}}_{s}$ the forcing terms. System (2.2) has to be endowed with suitable Dirichlet or Neumann boundary conditions on $\partial \Omega_{f}^{t} \backslash \Sigma^{t}$ and $\partial \Omega_{s}^{0} \backslash \Sigma^{0}$, and initial conditions in $\Omega^{0}$. Two transmission conditions are enforced at the interface: the continuity of fluid and structure velocities $(2.2)_{4}$ and the continuity of stresses $(2.2)_{5}$. The fluid and the structure are also coupled by the geometry problem, leading to a non-linear system of partial differential equations.

Remark 1. In the case of an incompressible structure, we have $\nu=0.5$. In this case, the Cauchy stress tensor is given by

$$
\boldsymbol{T}_{s}(\boldsymbol{\eta})=\lambda\left(\nabla \boldsymbol{\eta}+(\nabla \boldsymbol{\eta})^{T}\right)-\chi \boldsymbol{I},
$$

where $\chi$ is the structure pressure, and the structure equation $(2.2)_{3}$ becomes

$$
\begin{cases}\rho_{s} \frac{\partial^{2} \overline{\boldsymbol{\eta}}}{\partial t^{2}}-\nabla \cdot \overline{\boldsymbol{T}}_{s}=\overline{\boldsymbol{f}}_{s} & \text { in } \Omega_{s}^{0} \times(0, T), \\ \nabla \cdot \overline{\boldsymbol{\eta}}=0 & \text { in } \Omega_{s}^{0} \times(0, T) .\end{cases}
$$

2.1. Time discretization and FS interface treatment. Let us introduce the backward Euler time discretization for the fluid and the first order BDF scheme for the structure. The investigation of this work is independent on the time discretization, so that other schemes can be considered as well.

The main source of non-linearity comes from the fact that the interface (and hence the fluid domain) is unknown (geometrical non-linearity). This can be treated numerically in several ways. We focus here either on implicit treatments, where the FSI problem is solved at each time step by Picard, Newton or quasi-Newton iterations (see, e.g, $[11,19]$ ), or on explicit treatments, where the interface position is extrapolated from previous time steps. Similar considerations appy also to the non-linearity coming from the Navier-Stokes convective term.

Whatever strategy is adopted, a sequence of linearized FSI problems implicitly coupled through the interface conditions $(2.2)_{4,5}$ has to be solved. In particular, let us denote by $\Omega_{f}^{*}, \boldsymbol{u}^{*}$ and $\boldsymbol{w}^{*}$ the known fluid domain, fluid velocity and fluid domain velocity, where ${ }^{*}$ denotes either an extrapolated value (explicit algorithms) or 
the value of the previous subiteration (implicit algorithm). Moreover, let $g^{m}$ be the approximation of a function $g$ at time $t^{m}:=m \Delta t$, where $\Delta t$ is the time discretization parameter. Then, by applying the backward Euler scheme also for the continuity condition $(2.2)_{4}$, we obtain the following linear system

Given $\Omega_{f}^{*}, \boldsymbol{u}^{*}, \boldsymbol{w}^{*}, \boldsymbol{u}^{n}, \boldsymbol{\eta}^{n}$ and $\boldsymbol{\eta}^{n-1}$, find the fluid velocity $\boldsymbol{u}^{n+1}$, pressure $p^{n+1}$ and the structure displacement $\overline{\boldsymbol{\eta}}^{n+1}$ such that

$$
\begin{cases}\frac{\rho_{f}}{\Delta t} \boldsymbol{u}^{n+1}+\rho_{f}\left(\left(\boldsymbol{u}^{*}-\boldsymbol{w}^{*}\right) \cdot \nabla\right) \boldsymbol{u}^{n+1}-\nabla \cdot \boldsymbol{T}_{f}^{n+1}=\boldsymbol{f}_{f}^{n+1}+\frac{\rho_{f}}{\Delta t} \boldsymbol{u}^{n} & \text { in } \Omega_{f}^{*}, \\ \nabla \cdot \boldsymbol{u}^{n+1}=0 & \text { in } \Omega_{f}^{*}, \\ \frac{\rho_{s}}{\Delta t^{2}} \overline{\boldsymbol{\eta}}^{n+1}-\nabla \cdot \overline{\boldsymbol{T}}_{s}^{n+1}=\overline{\boldsymbol{f}}_{s}^{n+1}+\frac{\rho_{s}}{\Delta t^{2}}\left(2 \overline{\boldsymbol{\eta}}^{n}-\overline{\boldsymbol{\eta}}^{n-1}\right) & \text { in } \Omega_{s}^{0}, \\ \boldsymbol{u}=\frac{\boldsymbol{\eta}^{n+1}-\boldsymbol{\eta}^{n}}{\Delta t} & \text { on } \Sigma^{*}, \\ \boldsymbol{T}_{f}^{n+1} \boldsymbol{n}=\boldsymbol{T}_{s}^{n+1} \boldsymbol{n} & \text { on } \Sigma^{*},\end{cases}
$$

with suitable boundary conditions on the artificial sections.

For the sake of simplicity, here and in the sequel of the paper we drop the index ${ }^{n+1}$ referring to the time step.

2.2. Partitioned procedures. System (2.3) is still coupled through the interface conditions $(2.3)_{4}$ and $(2.3)_{5}$. In this work we consider schemes which guarantee strong enforcement of such conditions, thus achieving a perfect energy balance. In particular, we focus on the so called modular algorithms that involve separate fluid and structure solvers interacting through the exchange of suitable transmission conditions at the FS interface $\Sigma^{*}$. At convergence, they guarantee the continuity of the velocity and of the normal stress at $\Sigma^{*}$. In particular, an algorithm that uses an explicit treatment of the FS interface position and a strong coupling of the interface conditions is called semi-implicit (see $[5,10,20]$ ).

The most classical modular algorithm is the Dirichlet-Neumann (DN) scheme, which consists in solving iteratively the fluid problem with the structure velocity as Dirichlet boundary condition at the FS interface, and the structure problem with the fluid normal stress as Neumann boundary condition at $\Sigma^{*}$ (see, e.g., [19,21]). However, it has been shown in $[6,12,23]$ that in the presence of a large added mass effect, this procedure needs a strong relaxation and features a very slow convergence.

A new class of iterative procedures based on Robin transmission conditions, which generalizes the DN approach, has been introduced in [3]. In particular, the RobinRobin schemes are based on the following transmission conditions:

$$
\begin{aligned}
\alpha_{f} \boldsymbol{u}-\boldsymbol{T}_{f} \boldsymbol{n} & =\alpha_{f} \frac{\boldsymbol{\eta}-\boldsymbol{\eta}^{n}}{\Delta t}-\boldsymbol{T}_{s} \boldsymbol{n}, & & \text { on } \Sigma^{*}, \\
\frac{\alpha_{s}}{\Delta t} \boldsymbol{\eta}+\boldsymbol{T}_{s} \boldsymbol{n} & =\frac{\alpha_{s}}{\Delta t} \boldsymbol{\eta}^{n}+\alpha_{s} \boldsymbol{u}+\boldsymbol{T}_{f} \boldsymbol{n}, & & \text { on } \Sigma^{*},
\end{aligned}
$$

obtained by a linear combination of $(2.3)_{4}$ and $(2.3)_{5}$, with coefficients $\alpha_{f}, \alpha_{s}$ positive. We observe that with the choice $\alpha_{f} \rightarrow \infty$ and $\alpha_{s}=0$ we recover the DN scheme.

In this work we consider a fixed point algorithm consisting of subsequent iterations of fluid subproblems with interface condition $(2.4)_{1}$ and structure subproblems with interface condition $(2.4)_{2}$ :

Robin-Robin partitioned algorithm: Given $\Omega_{f}^{*}, \boldsymbol{u}^{*}, \boldsymbol{w}^{*}, \boldsymbol{u}^{n}, \boldsymbol{\eta}^{n}$ and $\boldsymbol{\eta}^{n-1}$ and the solution at previous iteration $\boldsymbol{\eta}_{m-1}$, find at each iteration $m$ the fluid velocity 
$\boldsymbol{u}_{m}$, the fluid pressure $p_{m}$ and the structure displacement $\overline{\boldsymbol{\eta}}_{m}$ until convergence, such that

Fluid problem:

$$
\begin{array}{ll}
\frac{\rho_{f}}{\Delta t} \boldsymbol{u}_{m}+\rho_{f}\left(\left(\boldsymbol{u}^{*}-\boldsymbol{w}^{*}\right) \cdot \nabla\right) \boldsymbol{u}_{m}-\nabla \cdot \boldsymbol{T}_{f, m}=\boldsymbol{f}_{f}+\frac{\rho_{f}}{\Delta t} \boldsymbol{u}^{n} & \text { in } \Omega_{f}^{*}, \\
\nabla \cdot \boldsymbol{u}_{m}=0 & \text { in } \Omega_{f}^{*}, \\
\alpha_{f} \boldsymbol{u}_{m}-\boldsymbol{T}_{f, m} \boldsymbol{n}=\alpha_{f} \frac{\boldsymbol{\eta}_{m-1}-\boldsymbol{\eta}^{n}}{\Delta t}-\boldsymbol{T}_{s, m-1} \boldsymbol{n}, & \text { on } \Sigma^{*},
\end{array}
$$

Structure problem

$$
\begin{array}{ll}
\frac{\rho_{s}}{\Delta t^{2}} \overline{\boldsymbol{\eta}}_{m}-\nabla \cdot \overline{\boldsymbol{T}}_{s, m}=\overline{\boldsymbol{f}}_{s}+\frac{\rho_{s}}{\Delta t^{2}}\left(2 \overline{\boldsymbol{\eta}}^{n}-\overline{\boldsymbol{\eta}}^{n-1}\right) & \text { in } \Omega_{s}^{0}, \\
\frac{\alpha_{s}}{\Delta t} \boldsymbol{\eta}_{m}+\boldsymbol{T}_{s, m} \boldsymbol{n}=\frac{\alpha_{s}}{\Delta t} \boldsymbol{\eta}^{n}+\alpha_{s} \boldsymbol{u}_{m}+\boldsymbol{T}_{f, m} \boldsymbol{n}, & \text { on } \Sigma^{*}
\end{array}
$$

This strategy can also be interpreted as a preconditioned Richardson algorithm applied to a suitable interface condition (see [2]).

3. Convergence analysis of simplified problems. In this Section we analyze the convergence of the RR scheme applied to three different reduced FSI problems, which are simple enough to be analyzed theoretically. On the other hand, they feature a behavior similar to the more complex system (2.3). Therefore, we expect that the theoretical results obtained from the analysis of these models give insightful information also for system (2.3). This analysis is performed by following the optimization strategy proposed in the Optimized Schwarz Methods literature (see $[1,13,14]$ for instance). In these works, a convergence analysis of two and three dimensional coupled problems with Robin trasmission conditions, based on the application of the Fourier transform, has been derived, for the Laplace, Helmoltz and Maxwell equations.

In order to apply this strategy to the FSI problem, we introduce suitable simplifying assumptions and reduced models. In particular, in all the convergence analysis, we consider a fixed FSI domain. The fluid domain is the half plane $\Omega_{f}=\{\boldsymbol{x}=(x, y) \in$ $\left.\mathbb{R}^{2}: x<0\right\}$, the FS interface the line $\Sigma=\left\{(x, y) \in \mathbb{R}^{2}: x=0\right\}$, the structure domain either coincides with the interface $\Sigma$ (generalized string model) or is the thick region $\Omega_{s}=\left[0, H_{s}\right] \times \mathbb{R}$ in the case of linear incompressibile elasticity. In all the cases considered, we allow the FS interface to move only in the normal direction.

We will base our convergence analysis on a Fourier transform in the direction tangential to the FS interface (corresponding to the $y$ variable in the case at hand), which is defined, for $w(x, y) \in L^{2}\left(\mathbb{R}^{2}\right)$, as

$$
\mathcal{F}: w(x, y) \mapsto \widehat{w}(x, k)=\int_{\mathbb{R}} e^{-i k y} w(x, y) d y,
$$

where $k$ is the frequency variable. We will then be able to quantify the error, in the frequency space, between the pressure at the $m$-th iteration, $\widehat{p}_{m}(x, k)$, and the exact pressure $\widehat{p}(x, k)$. This allows us to define, on the FS interface, the reduction factor at iteration $m$ for each frequency as

$$
\rho_{m}(k):=\frac{\left|\widehat{p}_{m}(0, k)-\widehat{p}(0, k)\right|}{\left|\widehat{p}_{m-1}(0, k)-\widehat{p}(0, k)\right|} .
$$

The RR algorithm converges if, at each iteration $m$, we have $\rho_{m}(k)<1$ for all the relevant frequencies of the problem, namely for $0 \leq k \leq k_{\max }$, where $k_{\max }$ is the maximal frequency supported by the numerical grid, and is of order $\pi / h$ ( $h$ being the mesh parameter). 
3.1. Potential flow - Generalized string model (P/GS). The first simplified model is obtained by considering a potential flow described by the Darcy equations for the fluid and the generalized string model for the structure (see [22])

$$
\begin{array}{lr}
\rho_{f} \delta_{t} \boldsymbol{u}+\nabla p=\mathbf{0} & \text { in } \Omega_{f}, \\
\nabla \cdot \boldsymbol{u}=0 & \text { in } \Omega_{f}, \\
\boldsymbol{u} \cdot \boldsymbol{n}=\delta_{t} \eta & \text { on } \Sigma, \\
p=\rho_{s} H_{s} \delta_{t t} \eta+\beta H_{s} \eta-G H_{s} \partial_{y y} \eta, & \text { on } \Sigma,
\end{array}
$$

where $G=K \lambda_{1} / 2$, with $K$ the Timoshenko correction factor, and we have set $\delta_{t} w:=$ $\frac{w-w^{n}}{\Delta t}, \delta_{t t} w:=\frac{\delta_{t} w-\delta_{t} w^{n}}{\Delta t}$, where $w$ could be a scalar or a vector function and as usual we have omitted the time index $n+1$. The reaction term $\beta$, arising in the derivation of the generalized string model in the $3 \mathrm{D}$ case, is introduced also in the $2 \mathrm{D}$ case to take into account for transversal membrane effects. Problem (3.1) has to be completed with initial conditions and with boundary conditions here reducing to the assumption of boundedness for $x \rightarrow-\infty$ and $|y| \rightarrow \infty$. We point out that the structure displacement is in the $x$ direction, which is the normal direction to the FS interface. The structure equation $(3.1)_{4}$ enforces the continuity of the normal stress at the interface along the normal direction. We observe that the analysis of this symplified problem has been already performed in [3]. For completeness, we report here the analysis via Fourier transform, which is functional for the further developments.

By combining linearly $(3.1)_{3}$ and $(3.1)_{4}$ with coefficients $\left(\alpha_{f},-1\right)$ and $\left(\alpha_{s}, 1\right)$, respectively, we obtain two Robin boundary conditions. Observe that in this problem the viscous terms have been neglected so the fluid Cauchy stress tensor reduces to the only pressure. By setting $u_{x}=\boldsymbol{u} \cdot \boldsymbol{n}$, with $\boldsymbol{n}$ the normal unit vector to the FS interface, and using coefficients $\left(\alpha_{f},-1\right)$, we obtain

$$
\alpha_{f} u_{x}-p=\alpha_{f} \delta_{t} \eta-\rho_{s} H_{s} \delta_{t t} \eta-\beta H_{s} \eta+G H_{s} \partial_{y y} \eta,
$$

that is

$$
\alpha_{f} \Delta t \delta_{t} u_{x}-p=\alpha_{f} \delta_{t} \eta-\rho_{s} H_{s} \delta_{t t} \eta-\beta H_{s} \eta+G H_{s} \partial_{y y} \eta-\alpha_{f} u_{x}^{n} .
$$

Then, the Robin transmission condition for the fluid problem can be rearranged as

$$
\alpha_{f} \Delta t \delta_{t} u_{x}-p=\left(\frac{\alpha_{f}}{\Delta t}-\frac{\rho_{s} H_{s}}{\Delta t^{2}}-\beta H_{s}\right) \eta+G H_{s} \partial_{y y} \eta+F_{1}\left(u_{x}^{n}, \eta^{n}, \eta^{n-1}\right),
$$

where $F_{1}\left(u_{x}^{n}, \eta^{n}, \eta^{n-1}\right)$ accounts for terms at previous time steps.

By using coefficients $\left(\alpha_{s}, 1\right)$, we have

$$
\rho_{s} H_{s} \delta_{t t} \eta+\beta H_{s} \eta-G H_{s} \partial_{y y} \eta+\alpha_{s} \delta_{t} \eta=p+\alpha_{s} u_{x},
$$

and the Robin condition for the structure problem can be rearranged as

$$
\left(\frac{\rho_{s} H_{s}}{\Delta t^{2}}+\beta H_{s}+\frac{\alpha_{s}}{\Delta t}\right) \eta-G H_{s} \partial_{y y} \eta=\alpha_{s} \Delta t \delta_{t} u_{x}+p+F_{2}\left(u^{n}, \eta^{n}, \eta^{n-1}\right),
$$

where again $F_{2}\left(u^{n}, \eta^{n}, \eta^{n-1}\right)$ accounts for terms at previous time steps.

Let $m$ be an iteration index. Then, the corresponding iterative Robin-Robin algorithm reads:

Given $\boldsymbol{u}^{0}, p^{0}, \eta^{0}$, solve for $m \geq 0$ 
1. Fluid problem

$$
\begin{aligned}
& \rho_{f} \delta_{t} \boldsymbol{u}_{m+1}+\nabla p_{m+1}=\mathbf{0} \quad \text { in } \Omega_{f}, \\
& \begin{array}{ll}
\nabla \cdot \boldsymbol{u}_{m+1}=0 & \text { in } \Omega_{f},
\end{array} \\
& \begin{aligned}
\alpha_{f} \Delta t \delta_{t} u_{x, m+1}-p_{m+1}= & \left(\frac{\alpha_{f}}{\Delta t}-\frac{\rho_{s} H_{s}}{\Delta t^{2}}-\beta H_{s}\right) \eta_{m}+ \\
& +G H_{s} \partial_{y y} \eta_{m}+F_{1}\left(u_{x}^{n}, \eta^{n}, \eta^{n-1}\right) \quad \text { on } \Sigma
\end{aligned}
\end{aligned}
$$

2. Structure problem

$$
\begin{aligned}
& \left(\frac{\rho_{s} H_{s}}{\Delta t^{2}}+\beta H_{s}+\frac{\alpha_{s}}{\Delta t}\right) \eta_{m+1}-G H_{s} \partial_{y y} \eta_{m+1}= \\
& =\alpha_{s} \Delta t \delta_{t} u_{x, m+1}+p_{m+1}+F_{2}\left(u_{x}^{n}, \eta^{n}, \eta^{n-1}\right)
\end{aligned}
$$

We have the following

Proposition 1. The reduction factor of iterations (3.4)-(3.5) is given by

$$
\rho^{m}(k)=\rho^{P / G S}(k)=\left|\frac{\frac{\rho_{s} H_{s}}{\Delta t}+\beta H_{s} \Delta t+G H_{s} \Delta t k^{2}-\alpha_{f}}{\frac{\rho_{s} H_{s}}{\Delta t}+\beta H_{s} \Delta t+G H_{s} \Delta t k^{2}+\alpha_{s}} \cdot \frac{\rho_{f}-\alpha_{s} \Delta t k}{\rho_{f}+\alpha_{f} \Delta t k}\right|,
$$

which is independent of the iteration $m$.

Moreover, for all Fourier modes $k \in\left[0, k_{\max }\right]$, there exist $\alpha_{f}^{\max }=\alpha_{f}^{\max }(k)$ and $\alpha_{s}^{\max }=\alpha_{s}^{\max }(k)$ such that, given $\boldsymbol{u}^{0}, p^{0}, \eta^{0}$, iterations (3.4)-(3.5) converge if $\alpha_{f} \in$ $\left[0, \alpha_{f}^{\max }\right)$ and $\alpha_{s} \in\left[0, \alpha_{s}^{\max }\right)$ and if at least one of the two values is different from 0.

Proof. Thanks to the relation

$$
\partial_{x} p=-\rho_{f} \delta_{t} u_{x}, \quad \text { on } \Sigma,
$$

obtained by restricting the first equation of the fluid problem $(3.4)_{1}$ on the FS interface, it is possible to rewrite the Robin interface conditions $(3.4)_{3}$ and (3.5) in terms of the sole pressure, obtaining, on $\Sigma$,

$$
\begin{aligned}
& \frac{\alpha_{f} \Delta t}{\rho_{f}} \partial_{x} p^{m+1}+p^{m+1}=\left(\frac{\rho_{s} H_{s}}{\Delta t^{2}}+\beta H_{s}-\frac{\alpha_{f}}{\Delta t}\right) \eta^{m}-G H_{s} \partial_{y y} \eta^{m}-F_{1}\left(u_{x}^{n}, \eta^{n}, \eta^{n-1}\right), \\
& \left(\frac{\rho_{s} H_{s}}{\Delta t^{2}}+\beta H_{s}+\frac{\alpha_{s}}{\Delta t}\right) \eta^{m}-G H_{s} \partial_{y y} \eta^{m}=p^{m}-\frac{\alpha_{s} \Delta t}{\rho_{f}} \partial_{x} p^{m}+F_{2}\left(u_{x}^{n}, \eta^{n}, \eta^{n-1}\right) .
\end{aligned}
$$

We point out that for the structure problem we wrote the $m$-th iteration instead of the $m+1$-th.

Since the problems involved are linear, we analyze without loss of generality the convergence to the zero solution when the forcing terms vanish, namely for $F_{1}\left(u_{x}^{n}, \eta^{n}, \eta^{n-1}\right)=F_{2}\left(u_{x}^{n}, \eta^{n}, \eta^{n-1}\right)=0$.

The divergence free condition on $\boldsymbol{u}$ allows us to rewrite the fluid problem in the unknown pressure

$$
\triangle p=0 \quad \text { in } \Omega_{f} .
$$

Applying the Fourier transform in the $y$ direction, we obtain the following ordinary differential equation for the pressure

$$
-\partial_{x x} \widehat{p}^{m+1}+k^{2} \widehat{p}^{m+1}=0 \quad \text { in }(-\infty, 0),
$$


whose solution is given by $\widehat{p}^{m+1}(x, k)=A(k) e^{k x}+B(k) e^{-k x}$. The boundedness assumption on the solution entails $B(k)=0$, thus

$$
\widehat{p}(x, k)=A(k) e^{k x},
$$

for a suitable $A(k)$.

Now, the interface conditions (3.8) become

$$
\begin{array}{ll}
\widehat{p}^{m+1}+\frac{\alpha_{f} \Delta t}{\rho_{f}} \partial_{x} \widehat{p}^{m+1}=\left(\frac{\rho_{s} H_{s}}{\Delta t^{2}}+\beta H_{s}+G H_{s} k^{2}-\frac{\alpha_{f}}{\Delta t}\right) \widehat{\eta}^{m}, & \text { for } x=0, \\
\left(\frac{\rho_{s} H_{s}}{\Delta t^{2}}+\beta H_{s}+G H_{s} k^{2}+\frac{\alpha_{s}}{\Delta t}\right) \widehat{\eta}^{m}=\widehat{p}^{m}-\frac{\alpha_{s} \Delta t}{\rho_{f}} \partial_{x} \widehat{p}^{m}, & \text { for } x=0 .
\end{array}
$$

By noticing that $\partial_{x} \widehat{p}^{m+1}=A^{m+1}(k) k e^{k x}$, we have

$$
\begin{array}{ll}
\left(\frac{\rho_{f}+\alpha_{f} \Delta t k}{\rho_{f}}\right) A^{m+1}(k)=\left(\frac{\rho_{s} H_{s}}{\Delta t^{2}}+\beta H_{s}+G H_{s} k^{2}-\frac{\alpha_{f}}{\Delta t}\right) \widehat{\eta}^{m}, & \text { for } x=0, \\
\left(\frac{\rho_{s} H_{s}}{\Delta t^{2}}+\beta H_{s}+G H_{s} k^{2}+\frac{\alpha_{s}}{\Delta t}\right) \widehat{\eta}^{m}=\frac{\rho_{f}-\alpha_{s} \Delta t k}{\rho_{f}} A^{m}(k), & \text { for } x=0 .
\end{array}
$$

We thus have $\left|A^{m+1}(k)\right|=\rho(k)\left|A^{m}(k)\right|$, with reduction factor $\rho$ given by (3.6).

In order to guarantee convergence, we have to find suitable values of $\alpha_{f}$ and $\alpha_{s}$ such that $\rho^{P / G S}<1$. To do this, if we consider the two factors $d_{1}$ and $d_{2}$ such that $\rho^{P / G S}=\left|d_{1} d_{2}\right|$ in (3.6), we obtain sufficient conditions by imposing that $\left|d_{1}\right|<1$ and $\left|d_{2}\right|<1$, separately. For the first term, $d_{1}=\frac{\frac{\rho_{s} H_{s}}{\Delta t}+\beta H_{s} \Delta t+G H_{s} \Delta t k^{2}-\alpha_{f}}{\frac{\rho_{s} H_{s}}{\Delta t}+\beta H_{s} \Delta t+G H_{s} \Delta t k^{2}+\alpha_{s}}$, it is sufficient to have $\alpha_{f} \in\left[0, \alpha_{f}^{\max }\right)$, with

$$
\alpha_{f}^{\max }(k)=2\left(\frac{\rho_{s} H_{s}}{\Delta t}+\beta H_{s} \Delta t+G H_{s} \Delta t k^{2}\right) .
$$

For the second term, $d_{2}=\frac{\rho_{f}-\alpha_{s} \Delta t k}{\rho_{f}+\alpha_{f} \Delta t k}$, it is sufficient to have $\alpha_{s} \in\left[0, \alpha_{s}^{\max }\right)$, where

$$
\alpha_{s}^{\max }(k)=\frac{2 \rho_{f}}{\Delta t k} .
$$

A less sharp upper bound, but independent of $k$, is given by

$$
\alpha_{s}^{\max }=\frac{2 \rho_{f}}{\Delta t k_{\max }} .
$$

Remark 2. We observe that function $A(k)$ is in general complex. However, in the particular case where the initial condition for the $x$ component of the fluid velocity is symmetric with respect to the $x$-axis, then its Fourier transform is real and therefore $A$ is real for each time (see (3.7)) and $u_{x}$ remains symmetric at each time.

Remark 3. For the DN scheme, that is for $\alpha_{f} \rightarrow \infty$ and $\alpha_{s}=0$, we obtain

$$
\rho^{P / G S}(k)=\left|\frac{\rho_{f}}{k\left(\rho_{s} H_{s}+\beta H_{s} \Delta t^{2}+G H_{s} \Delta t^{2} k^{2}\right)}\right| .
$$

Taking $k=0$, we have that no convergence of the DN scheme is achieved, even with relaxation $\left(\rho^{P / G S}(0) \rightarrow \infty\right)$. This is due to the unboundedness of the fluid domain. If 
we consider a bounded domain $\mathbb{R} \times[-L, 0]$, we have that $k \in\left(k_{\min }, k_{\max }\right)$, with $k_{\min }$ of the order of $\pi / L>0$ (see [6]). In this case, the reduction factor of the DN scheme is always bounded, but it highlights a high dependence of the convergence performances on the ratio $\rho_{f} / \rho_{s}$, as well on $k_{\text {min }}$. In particular, if $\rho_{f} / \rho_{s}$ is not small enough, for $k$ sufficiently small we have $\rho^{P / G S}(k)>1$, so that a relaxation is needed to reach convergence. On the contrary, for a general $R R$ scheme the sensitivity of $\rho^{P / G S}$ on the ratio $\rho_{f} / \rho_{s}$ is minimal and, as stated by Proposition 1, there exist always suitable values of $\alpha_{f}$ and $\alpha_{s}$ such that for each $k$ convergence is guaranteed without relaxation.

3.2. Stokes - Generalized string model (S/GS). Let us now consider the coupling between the unsteady Stokes problem for the fluid and the generalized string model for the structure. The fluid and structure domains are the same of the previous problem and again we consider only normal displacement of the FS interface.

In this case, the fluid stress tensor is given by $\boldsymbol{T}_{f}=p \boldsymbol{I}+\mu\left(\nabla \boldsymbol{u}+\nabla^{T} \boldsymbol{u}\right)$, since the viscous term is now considered and the normal component of the normal stress at the interface $\Sigma$ is $\boldsymbol{n}^{T} \cdot \boldsymbol{T}_{f} \boldsymbol{n}=-p+\mu \partial_{x} u_{x}$. Therefore, the Robin-Robin sequential algorithm for the generalized Stokes-generalized string model coupling is given by

1. Fluid problem

$$
\begin{aligned}
& \rho_{f} \delta_{t} \boldsymbol{u}^{m+1}-\mu \triangle \boldsymbol{u}^{m+1}+\nabla p^{m+1}=\mathbf{0} \quad \text { in } \Omega_{f} \\
& \nabla \cdot \boldsymbol{u}^{m+1}=0 \quad \text { in } \Omega_{f} \\
& \alpha_{f} u_{x}^{m+1}-p^{m+1}+\mu \partial_{x} u_{x}^{m+1}= \\
& =\left(\frac{\alpha_{f}}{\Delta t}-\frac{\rho_{s} H_{s}}{\Delta t^{2}}-\beta H_{s}\right) \eta^{m}+G H_{s} \partial_{y y} \eta^{m}+F_{1}\left(\eta^{n}, \eta^{n-1}\right) \quad \text { on } \Sigma
\end{aligned}
$$

2. Structure problem

$$
\begin{aligned}
& \left(\frac{\rho_{s} H_{s}}{\Delta t^{2}}+\beta H_{s}+\frac{\alpha_{s}}{\Delta t}\right) \eta^{m+1}-G H_{s} \partial_{y y} \eta^{m}= \\
& =\alpha_{s} u_{x}^{m+1}+p^{m+1}-\mu \partial_{x} u^{m+1}+F_{2}\left(\eta^{n}, \eta^{n-1}\right)
\end{aligned}
$$

where we have set $\left.u_{y}\right|_{\Sigma}=0$ and, again, $F_{1}$ and $F_{2}$ account for terms at previous time steps. We have the following

Proposition 2. The reduction factor of iterations (3.13)-(3.14) is given by

$$
\rho^{S / G S}(k)=\left|\frac{\frac{\rho_{s} H_{s}}{\Delta t}+\beta H_{s} \Delta t+G H_{s} \Delta t k^{2}-\alpha_{f}}{\frac{\rho_{s} H_{s}}{\Delta t}+\beta H_{s} \Delta t+G H_{s} \Delta t k^{2}+\alpha_{s}} \cdot \frac{\alpha_{s} k-\mu \gamma_{f}\left(k+\gamma_{f}\right)}{\alpha_{f} k+\mu \gamma_{f}\left(k+\gamma_{f}\right)}\right|,
$$

where

$$
\gamma_{f}(k)=\sqrt{\rho_{f} /(\mu \Delta t)+k^{2}}
$$

which is independent of the iteration $m$.

Moreover, for all Fourier modes $k \in\left[0, k_{\max }\right]$ there exist $\alpha_{f}^{\max }=\alpha_{f}^{\max }(k)$ and $\alpha_{s}^{\max }=\alpha_{s}^{\max }(k)$ such that, given $\boldsymbol{u}^{0}, p^{0}, \eta^{0}$, iterations (3.13)-(3.14) converge if $\alpha_{f} \in$ $\left[0, \alpha_{f}^{\max }\right)$ and $\alpha_{s} \in\left[0, \alpha_{s}^{\max }\right)$ and if at least one of the two values is different from 0. 
Proof. In order to write the fluid Robin interface condition $(3.13)_{3}$ in terms of the sole pressure, we need again to write the interface velocity $\boldsymbol{u}$ as a function of $p$. However, in this case we have

$$
\partial_{x} p=-\rho_{f} \delta_{t} u_{x}+\mu \triangle u_{x} \quad \text { on } \Sigma,
$$

so that the expression of $u_{x}$ at the interface in terms of $p$ is not straightforward anymore. However, by applying the divergence operator to the fluid problem $(3.13)_{1}$ we obtain again problem (3.9) for the pressure solely. We notice that the solution of this problem after applying the Fourier transform in the $y$ direction is again of the form (3.10), for a suitable $A(k)$, which in this case depends also on $\triangle u_{x}$.

Now, the fluid problem in the $x$ direction after the application of the Fourier transform in the $y$ direction, reads

$$
\frac{\rho_{f}}{\mu \Delta t} \widehat{u}_{x}-\partial_{x x} \widehat{u}_{x}+k^{2} \widehat{u}_{x}+\frac{A}{\mu} k e^{k x}=\frac{\rho_{f}}{\mu \Delta t} \widehat{u}_{x}^{n}, \quad \text { in } \Omega_{f},
$$

having noticed that $\partial_{x} \widehat{p}=k A e^{k x}$. The homogeneous solutions of this equation are $\widehat{u}_{x, 1}^{o}(x, k)=B(k) e^{\gamma_{f} x}$ and $\widehat{u}_{x, 2}^{o}(x, k)=C(k) e^{-\gamma_{f} x}$, for suitable $B$ and $C$ and where $\gamma_{f}$ is given by (3.16). From the boundedness assumption, it follows $C \equiv 0$. As before, in the convergence analysis the terms at the previous time steps are discarded, so that we drop them from now on. As particular solution let us consider $\widehat{u}_{x}^{p}(x, k)=$ $-\frac{k A}{2 \mu \gamma_{f}\left(k-\gamma_{f}\right)} e^{\gamma_{f} x}+\frac{k A}{\mu\left(k+\gamma_{f}\right)\left(k-\gamma_{f}\right)} e^{k x}$. Adding this solution to the homogeneous ones, we obtain

$$
\widehat{u}_{x}(x, k)=\left(B-\frac{k A}{2 \mu \gamma_{f}\left(k-\gamma_{f}\right)}\right) e^{\gamma_{f} x}+\frac{k A}{\mu\left(k+\gamma_{f}\right)\left(k-\gamma_{f}\right)} e^{k x} .
$$

The equation for $u_{y}$ reads

$$
\frac{\rho_{f}}{\mu \Delta t} \widehat{u}_{y}-\partial_{x x} \widehat{u}_{y}+k^{2} \widehat{u}_{y}-i k \frac{A}{\mu} e^{k x}=\frac{\rho_{f}}{\mu \Delta t} \widehat{u}_{y}^{n}, \quad \text { in } \Omega_{f},
$$

having noticed that $\partial_{y} \widehat{p}=-i k A e^{k x}$. Then, as before, it is possible to show that the solution is given by

$$
\widehat{u}_{y}(x, k)=\left(D+\frac{i k A}{2 \mu \gamma_{f}\left(k-\gamma_{f}\right)}\right) e^{\gamma_{f} x}-\frac{i k A}{\mu\left(k+\gamma_{f}\right)\left(k-\gamma_{f}\right)} e^{k x}
$$

for a suitable $D(k)$.

From the incompressibility constraint, we have $\partial_{x} \widehat{u}_{x}-i k \widehat{u}_{y}=0$, obtaining

$$
\left(\gamma_{f} B-i k D+\frac{k A}{2 \mu \gamma_{f}}\right) e^{\gamma_{f} x}=0
$$

and then

$$
\gamma_{f} B-i k D+\frac{k A}{2 \mu \gamma_{f}}=0 .
$$

From the interface condition for the velocity $u_{y}$, namely $u_{y}=0$ for $x=0$, we obtain

$$
\widehat{u}_{y}(0, k)=D+\frac{i k A}{2 \mu \gamma_{f}\left(k-\gamma_{f}\right)}-\frac{i k A}{\mu\left(k+\gamma_{f}\right)\left(k-\gamma_{f}\right)}=0
$$


and then $D=-\frac{i k A}{2 \mu \gamma_{f}\left(k+\gamma_{f}\right)}$, which inserted in (3.19) leads to

$$
\gamma_{f} B+\frac{k A}{2 \mu\left(k+\gamma_{f}\right)}=0
$$

Then, the normal stress at the FS interface in the $x$ direction is

$$
\left.\left(\widehat{p}-\mu \partial_{x} \widehat{u}_{x}\right)\right|_{x=0}=A-\mu\left(\gamma_{f} B-\frac{k A}{2 \mu\left(k-\gamma_{f}\right)}+\frac{k^{2} A}{\mu\left(k+\gamma_{f}\right)\left(k-\gamma_{f}\right)}\right)=A
$$

where the last equality is obtained thanks to (3.20).

Let us finally compute the value of the velocity in the $x$ direction at the FS interface:

$$
\left.\widehat{u}_{x}\right|_{x=0}=B-\frac{k A}{2 \mu \gamma_{f}\left(k-\gamma_{f}\right)}+\frac{k A}{\mu\left(k+\gamma_{f}\right)\left(k-\gamma_{f}\right)}=-\frac{k A}{\mu \gamma_{f}\left(k+\gamma_{f}\right)},
$$

where the last equality is obtained again thanks to (3.20).

Therefore, owing to (3.21) and (3.22), the interface Robin conditions $(3.13)_{3}$ and (3.14) read

$$
\begin{array}{ll}
-\frac{\alpha_{f} k A^{m+1}}{\mu \gamma_{f}\left(k+\gamma_{f}\right)}-A^{m+1}=\left(-\frac{\rho_{s} H_{s}}{\Delta t^{2}}-\beta H_{s}-G H_{s} k^{2}+\frac{\alpha_{f}}{\Delta t}\right) \widehat{\eta}^{m}, & \text { for } x=0, \\
\left(\frac{\rho_{s} H_{s}}{\Delta t^{2}}+\beta H_{s}+G H_{s} k^{2}+\frac{\alpha_{s}}{\Delta t}\right) \widehat{\eta}^{m}=-\frac{\alpha_{s} k A^{m}}{\mu \gamma_{f}\left(\gamma_{f}+k\right)}+A^{m}, & \text { for } x=0 .
\end{array}
$$

We thus have $\left|A^{m+1}(k)\right|=\rho(k)\left|A^{m}(k)\right|$, where the reduction factor $\rho$ is given by (3.15).

In order to guarantee convergence, we have to find suitable values of $\alpha_{f}$ and $\alpha_{s}$ such that $\rho^{S / G S}<1$. Again, to do this, we obtain sufficient conditions by imposing that the two factors in (3.15) are separately less than 1 . The first term is equal to the previous analysis so that the upper bound $\alpha_{f}^{\max }$ is given again by (3.11). The second term is $d_{2}=\frac{\alpha_{s} k-\mu \gamma_{f}\left(k+\gamma_{f}\right)}{\alpha_{f} k+\mu \gamma_{f}\left(k+\gamma_{f}\right)}$, so that it is sufficient to take as upper bound

$$
\alpha_{s}^{\max }(k)=\frac{2 \mu \gamma_{f}\left(k+\gamma_{f}\right)}{k}
$$

A less sharp upper bound, but independent of $k$, is given by

$$
\alpha_{s}^{\max }=\frac{2 \mu \gamma_{f}^{*}\left(k^{*}+\gamma_{f}^{*}\right)}{k^{*}}
$$

where $\gamma_{f}^{*}=\gamma_{f}\left(k^{*}\right)$ and $k^{*}=\sqrt{\frac{(\sqrt{5}-1) \rho_{f}}{2 \mu \Delta t}}$ is such that $\alpha_{s}^{\max }$ in (3.23) is minimal.

Remark 4. We point out that functions $A(k), B(k)$ and $D(k)$ are in general complex. In particular, if the initial condition for the $x$ component of the fluid velocity is symmteric with respect to the $x$-axis, $A$ and $B$ are real. In this case (3.17) has real coefficients so that $u_{x}$ is symmetric for each time. Conversely, if the initial condition for the $y$ component of the fluid velocity is emi-symmteric with respect to the $x$-axis, then equation (3.18) has imaginary coefficients, so that D has to be imaginary and $u_{y}$ is emi-symmetric for each time. This is not surprising, since in the $y$ direction we have two waves propagating in the two opposite directions. 
Remark 5. For the DN scheme we obtain

$\rho^{S / G S}=\left|\frac{\mu \gamma_{f}\left(k+\gamma_{f}\right) \Delta t}{k\left(\rho_{s} H_{s}+\beta H_{s} \Delta t^{2}+G H_{s} \Delta t^{2} k^{2}\right)}\right|=\left|\frac{\rho_{f}+k^{2} \mu \Delta t+\sqrt{\rho_{f} \mu k^{2} \Delta t+\mu^{2} k^{4} \Delta t^{2}}}{k\left(\rho_{s} H_{s}+\beta H_{s} \Delta t^{2}+G H_{s} \Delta t^{2} k^{2}\right)}\right|$

which highlights again the high dependence of the convergence performances on the ratio $\rho_{f} / \rho_{s}$. Also in this case, for a general $R R$ scheme the sensitivity of $\rho^{S / G S}$ on this ratio is minimal.

3.3. Stokes - Linear incompressibile elasticity. Let us consider a thick structure, whose thickness is $H_{s}$. Again, the structure domain is unbounded in the $y$ direction, so that $\Omega_{s}=\left[0, H_{s}\right] \times \mathbb{R}$. We assume that the structure is incompressible. This is an assumption verified in many applications, for example in haemodynamics. Moreover, as in the previous problems, we set $\left.u_{y}\right|_{\Sigma}=0$, that is we allow the FS interface to move only in the normal direction. This assumption is not realistic in many practical applications, but, in the case of haemodynamics, which inspired the present work, the transversal displacement is typically much smaller than the normal one. Moreover, as in the generalized string model, to emulate a "cylindrically-shaped" structure and take into account for transversal membrane effects, we add a reaction term with coefficient $\beta$.

Therefore, the (discretized in time) structure problem reduces to a generalized Stokes problem and the $\mathrm{RR}$ algorithm reads:

1. Fluid problem

$$
\begin{array}{ll}
\rho_{f} \delta_{t} \boldsymbol{u}^{m+1}-\mu \triangle \boldsymbol{u}^{m+1}+\nabla p^{m+1}=\mathbf{0}, & \text { in } \Omega_{f}, \\
\nabla \cdot \boldsymbol{u}^{m+1}=0, & \text { in } \Omega_{f}, \\
\alpha_{f} u_{x}^{m+1}-p^{m+1}+\mu \partial_{x} u_{x}^{m+1}=\frac{\alpha_{f}}{\Delta t} \eta_{x}^{m}-\chi^{m}+\lambda \partial_{x} \eta_{x}^{m}+F_{1}\left(\eta_{x}^{n}, \eta_{x}^{n-1}\right), & \text { on } \Sigma .
\end{array}
$$

2. Structure problem

$$
\begin{array}{lc}
\rho_{s} \delta_{t t} \boldsymbol{\eta}^{m}-\lambda \triangle \boldsymbol{\eta}^{m}+\beta \boldsymbol{\eta}^{m}+\nabla \chi^{m}=\mathbf{0}, & \text { in } \Omega_{s}, \\
\nabla \cdot \boldsymbol{\eta}^{m}=0, & \text { in } \Omega_{s}, \\
\frac{\alpha_{s}}{\Delta t} \eta_{x}^{m}+\chi^{m}-\lambda \partial_{x} \eta_{x}^{m}=\alpha_{s} u_{x}^{m+1}+p^{m+1}-\mu \partial_{x} u_{x}^{m+1}+F_{2}\left(\eta_{x}^{n}, \eta_{x}^{n-1}\right), & \text { on } \Sigma,
\end{array}
$$

where $\chi$ is the pressure for the structure problem and we have set $\left.u_{y}\right|_{\Sigma}=\left.\eta_{y}\right|_{\Sigma}=0$. We have the following

Proposition 3. The reduction factor of iterations (3.25)-(3.26) is given by

$$
\rho^{S / S}(k)=\left|\frac{2 \alpha_{f} \delta-\varepsilon \Delta t-\Delta t}{2 \alpha_{s} \delta+\varepsilon \Delta t+\Delta t} \cdot \frac{\alpha_{s} k-\mu \gamma_{f}\left(k+\gamma_{f}\right)}{\alpha_{f} k+\mu \gamma_{f}\left(k+\gamma_{f}\right)}\right|
$$

where $\gamma_{f}$ is given by (3.16), and

$$
\begin{aligned}
& \varepsilon(k)=-1+\frac{2 k \gamma_{s}^{2}}{k^{2}-\gamma_{s}^{2}} H_{s}-\frac{2 k^{2} \gamma_{s}^{4}}{\left(k^{2}-\gamma_{s}^{2}\right)^{2}} H_{s}^{2}+O\left(H_{s}^{3}\right), \\
& \delta(k)=\frac{(\varepsilon(k)+1)}{2 \lambda \gamma_{s}}\left(\frac{1}{\gamma_{s} H_{s}}-\frac{\gamma_{s} H_{s}}{6}\right)^{2}+O\left(H_{s}^{2}\right)=\frac{k}{\lambda\left(k^{2}-\gamma_{s}^{2}\right)}\left(1-\frac{k \gamma_{s}^{2} H_{s}}{k^{2}-\gamma_{s}^{2}}\right)+O\left(H_{s}^{2}\right), \\
& \gamma_{s}(k)=\sqrt{\rho_{s} /\left(\lambda \Delta t^{2}\right)+\beta / \lambda+k^{2}} .
\end{aligned}
$$


Moreover, for all Fourier modes $k \in\left[0, k_{\max }\right]$ there exist $\alpha_{f}^{\max }=\alpha_{f}^{\max }(k)$ and $\alpha_{s}^{\max }=\alpha_{s}^{\max }(k)$ such that, given $\boldsymbol{u}^{0}, p^{0}, \eta^{0}$, iterations (3.25)-(3.26) converge if $\alpha_{f} \in$ $\left[0, \alpha_{f}^{\max }\right)$ and $\alpha_{s} \in\left[0, \alpha_{s}^{\max }\right)$ and if at least one of the two values is different from 0 .

Proof. Let us apply the divergence operator to the structure equation $(3.26)_{1}$, obtaining again a problem for the pressure solely:

$$
\triangle \chi=0, \quad \text { in } \Omega_{s},
$$

with suitable boundary conditions depending on the value of $\boldsymbol{\eta}$. Again, we apply the Fourier transform in the $y$ direction, so that the solution can be written as

$$
\widehat{\chi}(x, k)=E(k) e^{k x}+F(k) e^{-k x}, \quad x>0,
$$

for suitable functions $E$ and $F$. In this case it is not possible anymore to exploit a boundedness assumption at infinity, so that both terms in (3.29) have to be considered.

Applying the Fourier transform along the $y$ direction to the first component of the structure problem $(3.26)_{1}$, we obtain

$$
\frac{\rho_{s}}{\lambda \Delta t^{2}} \widehat{\eta}_{x}-\partial_{x x} \widehat{\eta}_{x}+k^{2} \widehat{\eta}_{x}+\frac{\beta}{\lambda} \widehat{\eta}_{x}+\frac{k E}{\lambda} e^{k x}-\frac{k F}{\lambda} e^{-k x}=0, \quad x>0
$$

having noticed that $\partial_{x} \widehat{\chi}=k E e^{k x}-k F e^{-k x}$. The solution of this problem is given by

$$
\begin{gathered}
\widehat{\eta}_{x}(x, k)=\frac{k E(k)}{\lambda\left(k^{2}-\gamma_{s}^{2}\right)} e^{k x}-\frac{k F(k)}{\lambda\left(k^{2}-\gamma_{s}^{2}\right)} e^{-k x}+\left(L(k)-\frac{k E(k)}{2 \lambda \gamma_{s}\left(k-\gamma_{s}\right)}-\frac{k F(k)}{2 \lambda \gamma_{s}\left(k+\gamma_{s}\right)}\right) e^{\gamma_{s} x}+ \\
+\left(M(k)+\frac{k E(k)}{2 \lambda \gamma_{s}\left(k+\gamma_{s}\right)}+\frac{k F(k)}{2 \lambda \gamma_{s}\left(k-\gamma_{s}\right)}\right) e^{-\gamma_{s} x}
\end{gathered}
$$

where $\gamma_{s}$ is given by $(3.28)_{3}$ and $L(k)$ and $M(k)$ have to be properly determined.

The equation for $\widehat{\eta}_{y}$ reads

$$
\frac{\rho_{s}}{\lambda \Delta t^{2}} \widehat{\eta}_{y}-\partial_{x x} \widehat{\eta}_{y}+k^{2} \widehat{\eta}_{y}+\frac{\beta}{\lambda} \widehat{\eta}_{y}-\frac{i k E}{\lambda} e^{k x}-\frac{i k F}{\lambda} e^{-k x}=0, \quad x>0
$$

having noticed that $\partial_{y} \widehat{\chi}=-i k E e^{k x}-i k F e^{-k x}$. The solution of this problem is given by

$$
\begin{gathered}
\widehat{\eta}_{y}(x, k)=\frac{-i k E(k)}{\lambda\left(k^{2}-\gamma_{s}^{2}\right)} e^{k x}-\frac{i k F(k)}{\lambda\left(k^{2}-\gamma_{s}^{2}\right)} e^{-k x}+\left(N(k)+\frac{i k E(k)}{2 \lambda \gamma_{s}\left(k-\gamma_{s}\right)}-\frac{i k F(k)}{2 \lambda \gamma_{s}\left(k+\gamma_{s}\right)}\right) e^{\gamma_{s} x}+ \\
+\left(P(k)-\frac{i k E(k)}{2 \lambda \gamma_{s}\left(k+\gamma_{s}\right)}+\frac{i k F(k)}{2 \lambda \gamma_{s}\left(k-\gamma_{s}\right)}\right) e^{-\gamma_{s} x}
\end{gathered}
$$

where $N(k)$ and $P(k)$ are suitable functions of $k$.

Let us impose the incompressibility constraint $\partial_{x} \widehat{\eta}_{x}-i k \widehat{\eta}_{y}=0$. We obtain

$$
\left(\gamma_{s} L-i k N+\frac{k E}{2 \lambda \gamma_{s}}-\frac{k F}{2 \lambda \gamma_{s}}\right) e^{\gamma_{s} x}+\left(-\gamma_{s} M-i k P-\frac{k E}{2 \lambda \gamma_{s}}+\frac{k F}{2 \lambda \gamma_{s}}\right) e^{-\gamma_{s} x}=0
$$


This equation has to be satisfied for all $x>0$, so that it leads to the following conditions

$$
\begin{aligned}
& \gamma_{s}(L-M)-i k(N+P)=0 \\
& \gamma_{s} L-i k N+\frac{k(E-F)}{2 \lambda \gamma_{s}}=0
\end{aligned}
$$

Let us now impose that the velocity in the $y$ direction at the FS interface is zero. From (3.31), we obtain

$$
\left.\widehat{\eta}_{y}\right|_{x=0}=N+P=0,
$$

so that from $(3.32)_{1}$ we have $L=M$.

We observe that in the expressions of $\widehat{\eta}_{x}$ and $\widehat{\eta}_{y}$ in (3.30) and (3.31) we do not rely on any boundedness assumption, since the structure domain in bounded in the $x$ direction. However, we can prescribe that the normal stress at the outlet $x=H_{s}$ is zero. In particular, we have

$$
\begin{aligned}
& \left.\left(\lambda \partial_{x} \widehat{\eta}_{x}-\widehat{\chi}\right)\right|_{x=H_{s}}=0 \\
& \left.\frac{\lambda}{2}\left(\partial_{x} \widehat{\eta}_{y}-i k \widehat{\eta}_{x}\right)\right|_{x=H_{s}}=0 .
\end{aligned}
$$

The first condition allows to give a relation among $E, F$ and $L$, namely

$$
\begin{gathered}
L=\frac{2 \gamma_{s}^{2} E e^{k H_{s}}+2 \gamma_{s}^{2} F e^{-k H_{s}}-\left(k E\left(k+\gamma_{s}\right)+k F\left(k-\gamma_{s}\right)\right) e^{\gamma_{s} H_{s}}}{2 \lambda \gamma_{s}\left(k^{2}-\gamma_{s}^{2}\right)\left(e^{-\gamma_{s} H_{s}}-e^{\gamma_{s} H_{s}}\right)}+ \\
-\frac{\left(k E\left(k-\gamma_{s}\right)+k F\left(k+\gamma_{s}\right)\right) e^{-\gamma_{s} H_{s}}}{2 \lambda \gamma_{s}\left(k^{2}-\gamma_{s}^{2}\right)\left(e^{-\gamma_{s} H_{s}}-e^{\gamma_{s} H_{s}}\right)}=\frac{E+F}{2 \lambda \gamma_{s}}\left(\frac{1}{\gamma_{s} H_{s}}-\frac{\gamma_{s} H_{s}}{6}\right)+O\left(H_{s}^{2}\right) .
\end{gathered}
$$

The second condition allows to write $N$ as function of $E$ and $F$, namely

$$
\begin{aligned}
N=-\frac{i k L}{\gamma_{s}} & +\frac{1}{\gamma_{s}\left(e^{\gamma_{s} H_{s}}+e^{-\gamma_{s} H_{s}}\right)}\left[\frac{2 i k^{2} E}{\lambda\left(k^{2}-\gamma_{s}^{2}\right)} e^{k H_{s}}-\frac{2 i k^{2} F}{\lambda\left(k^{2}-\gamma_{s}^{2}\right)} e^{-k H_{s}}+\right. \\
& -\frac{i k}{2 \lambda \gamma_{s}\left(k^{2}-\gamma_{s}^{2}\right)}\left(\left(k+\gamma_{s}\right)^{2} E+\left(k-\gamma_{s}\right)^{2} F\right) e^{\gamma_{s} H_{s}}+ \\
& \left.+\frac{i k}{2 \lambda \gamma_{s}\left(k^{2}-\gamma_{s}^{2}\right)}\left(\left(k-\gamma_{s}\right)^{2} E+\left(k+\gamma_{s}\right)^{2} F\right) e^{-\gamma_{s} H_{s}}\right]= \\
= & -\frac{i k}{2 \lambda \gamma_{s}^{2}}(E+F)\left(\frac{1}{\gamma_{s} H_{s}}-\frac{\gamma_{s} H_{s}}{6}+\frac{k-\gamma_{s}}{k+\gamma_{s}} k H_{s}\right)+O\left(H_{s}^{2}\right) .
\end{aligned}
$$

This expression, together with $(3.32)_{2}$ allows to derive a relation between $E$ and $F$, namely

$$
F(k)=\varepsilon(k) E(k),
$$

with $\varepsilon$ given by $(3.28)_{1}$. From $(3.33)$, we have $L(k)=\delta(k) E(k)$, with $\delta$ given by $(3.28)_{2}$ 
Let us compute the values of the displacement and of the normal stress at the FS interface. From (3.30), we obtain

$$
\left.\widehat{\eta}_{x}\right|_{x=0}=2 L=2 \delta E
$$

and from (3.29) and (3.30)

$$
\left.\left(\lambda \partial_{x} \widehat{\eta}_{x}-\widehat{\chi}\right)\right|_{x=0}=-E-F=-(1+\varepsilon) E .
$$

The fluid problem is identical to the one analyzed in the previous section (Stokes/generalized string). We have therefore

$$
\begin{array}{lll}
p-\mu \partial_{x} \widehat{u}_{x}=A & \text { for } & x=0, \\
\widehat{u}_{x}=-\frac{{ }_{k A}}{\mu_{J}(k+\gamma)} & \text { for } & x=0 .
\end{array}
$$

Then the Robin interface conditions $(3.25)_{3}$ and $(3.26)_{3}$ read

$$
\begin{array}{rlr}
-\frac{\alpha_{f} k}{\mu \gamma_{f}\left(k+\gamma_{f}\right)} A^{m+1}-A^{m+1} & =\left(\frac{2 \alpha_{f} \delta}{\Delta t}-\varepsilon-1\right) E^{m} & \text { for } x=0, \\
\left(\frac{2 \alpha_{s} \delta}{\Delta t}+\varepsilon+1\right) E^{m} & =-\frac{\alpha_{s} k}{\mu \gamma_{f}\left(k+\gamma_{f}\right)} A^{m}+A^{m} & \text { for } x=0,
\end{array}
$$

and the reduction factor is given by (3.27).

In order to guarantee convergence, we have to find suitable values of $\alpha_{f}$ and $\alpha_{s}$ such that $\rho^{S / G S}<1$. Again, to do this, we obtain sufficient conditions by imposing that the two factors in (3.27) are separately less than 1. The second term is equal to the previous analysis so that the upper bound $\alpha_{s}^{\max }$ is given again by (3.23). The first term is $d_{1}=\frac{2 \alpha_{f} \delta-\varepsilon \Delta t-\Delta t}{2 \alpha_{s} \delta+\varepsilon \Delta t+\Delta t}$, so that it is sufficient to take as upper bound

$$
\alpha_{f}^{\max }(k)=\frac{(\varepsilon+1) \Delta t}{\delta} .
$$

Remark 6. For the DN scheme we obtain

$$
\rho^{S / S}=\left|\frac{2 \delta \mu \gamma_{f}\left(k+\gamma_{f}\right)}{k \Delta t(\varepsilon+1)}\right|=\left|\frac{\mu \gamma_{f}\left(k+\gamma_{f}\right)}{\lambda \gamma_{s}^{2} H_{s} k \Delta t}\right|=\left|\frac{a_{1} \rho_{f}+a_{2} \sqrt{\rho_{f}}+a_{3}}{a_{4} \rho_{s}+a_{5}}\right|,
$$

for suitable $a_{j}=a_{j}\left(k, \mu, \lambda, \beta, H_{s}, \Delta t\right), j=1, \ldots, 5$, and again the DN scheme highlights a high dependence of the convergence performances on the ratio $\rho_{f} / \rho_{s}$. In particular, we point out that $\lim _{\Delta t \rightarrow 0} \rho^{S / S}=\rho_{f} /\left(k \rho_{s} H_{s}\right)$. Also in this case, for a general $R R$ scheme the sensitivity of $\rho^{S / S}$ on this ratio is minimal.

4. Optimization of $\alpha_{f}$ and $\alpha_{s}$. With the aim of constructing RR schemes with good convergence properties, the problem of determining suitable values for parameters $\alpha_{f}$ and $\alpha_{s}$ has to be addressed. Indeed, the convergence performances of RR schemes heavily depend on this choice (see, in a different context, [15]). By maximizing the convergence rate of the corresponding algorithm, it is possible to determine either optimal or optimized values of the coefficients $\alpha_{f}$ and $\alpha_{s}$ in the Robin interface conditions. The classical approach in the Optimized Schwarz Methods literature consists in looking for parameters that, if possible, annihilate identically the reduction factor, ensuring convergence for the scheme in just two iterations for a twodomain decomposition. Such parameters are referred to as optimal. In particular, in a two-domain decomposition framework, we recall that the optimal interface conditions 
are of Robin type, where the Dirchlet-to-Neumann operator of one domain is applied as a boundary condition for the other domain. When such approach is not viable, the idea is to look for parameters that minimize the reduction factor.

In this section we look for optimized values for $\alpha_{f}$ and $\alpha_{s}$ either $k$-independent $\alpha_{f}=\alpha_{f, M}, \alpha_{s}=\alpha_{s, M}$, which lead to standard Robin boundary conditions (with standard mass matrix at the interface), or $k$ - dependent values of the form $\alpha_{f}=$ $\alpha_{f, M}+\alpha_{f, K} k^{2}, \alpha_{s}=\alpha_{s, M}+\alpha_{s, K} k^{2}$, which correspond to generalized Robin boundary conditions on $\Sigma$

$$
\begin{gathered}
\alpha_{f, M} \boldsymbol{u}-\alpha_{f, K} \triangle^{\Sigma} \boldsymbol{u}+\boldsymbol{T}_{f} \boldsymbol{n}=\alpha_{f, M}\left(\frac{\boldsymbol{\eta}-\boldsymbol{\eta}^{n}}{\Delta t}\right)-\alpha_{f, K} \triangle^{\Sigma}\left(\frac{\boldsymbol{\eta}-\boldsymbol{\eta}^{n}}{\Delta t}\right)+\boldsymbol{T}_{s} \boldsymbol{n}, \quad \text { (4.1) } \\
\frac{\alpha_{s, M}}{\Delta t} \boldsymbol{\eta}-\alpha_{s, K} \triangle^{\Sigma} \boldsymbol{\eta}+\boldsymbol{T}_{s} \boldsymbol{n}=\frac{\alpha_{s, M}}{\Delta t} \boldsymbol{\eta}^{n}-\alpha_{s, K} \triangle^{\Sigma} \boldsymbol{\eta}^{n}+\alpha_{s, M} \boldsymbol{u}-\alpha_{s, K} \triangle^{\Sigma} \boldsymbol{u}^{n}+\boldsymbol{T}_{f} \boldsymbol{n},
\end{gathered}
$$

where $\triangle^{\Sigma}$ is the Laplace-Beltrami operator over the interface $\Sigma$.

Before presenting the optimization procedures, we recall in the next subsection the choices proposed in [3] for a heuristic determination of the parameters.

4.1. Determination of $\alpha_{f}$ and $\alpha_{s}$ by heuristic methods. In [20] a membrane model has been proposed to describe in an approximated way the behaviour of thin structures. It is possible to "insert" the membrane equation into the fluid one as a boundary term (we highlight that in this case the structure domain coincides with the FS interface). This leads to a fluid problem with a suitable Robin boundary condition at the FS interface, which accounts for the structure (see [20]). In [2,3], this formulation has been used to obtain a plausible value of $\alpha_{f}$ in RR schemes, namely

$$
\alpha_{f}^{\text {heur }}=\frac{\rho_{s} H_{s}}{\Delta t}+\beta H_{s} \Delta t
$$

where $H_{s}$ is the structure thickness and

$$
\beta=\frac{E}{1-\nu^{2}}\left(4 \rho_{1}^{2}-2(1-\nu) \rho_{2}\right),
$$

with $\rho_{1}$ and $\rho_{2}$ the mean and Gaussian curvatures of the FS interface, respectively. We point out that $\alpha_{f}^{\text {heur }}$ is a function of the position on the interface.

RN schemes with $\alpha_{f}$ given by (4.3) exhibit very good performances for a wide range of added mass and are by far more efficient than DN strategies (see [2,3]). In particular, the theoretical analysis presented in these works, have shown that RN schemes with $\alpha_{f}^{\text {heur }}$ are less sensitive than DN schemes to the added-mass effect. Moreover, numerical experiments with high added-mass effect, highlighted that the RN scheme converges without any relaxation and faster than the DN scheme. However, in [2] $\mathrm{RN}$ has been shown to be sensitive to the choice of $\alpha_{f}$, leading to a deterioration of the performances when the curvature of the FS interface could not be computed accurately. On the contrary, the RN-GMRES scheme proposed in [2] is more robust with respect to the choice of $\alpha_{f}$.

For what concernes the parameter $\alpha_{s}$, in [3] it has been proved that the operator describing the added mass effect on the structure is not algebraic and its approximation by an algebraic relationship is not evident. In particular a generalized Robin condition (that involvs differential operators) is obtained with $\alpha_{s}=\left(\rho_{f} / \Delta t^{2}\right) \mathcal{M}(\cdot)$, where $\mathcal{M}$ is the added-mass operator, that is the Neumann-to-Dirichlet map (see $[3,6]$ ). In 
order to obtain a "classical" Robin condition, in [3] it has been proposed to approximate the operator $\mathcal{M}$ by $\theta \mu_{\max } I$, where $\mu_{\max }$ is the maximum eigenvalue of the added-mass operator, $I$ is the identity operator and $\theta$ is a coefficient suitably chosen, getting

$$
\alpha_{s}^{\text {heur }}=\theta \frac{\rho_{f} \mu_{\max }}{\Delta t}
$$

The drawback of this choice is that the scaling factor $\theta$ has to be tuned "by hand" to obtain good convergence properties. The numerical experiments in [3] showed that the tuned value $\theta$ seems to be very robust and practically independent of $\rho_{f}, \Delta t$ and of some geometrical parameters defining the physical domain (and then $\mu_{\max }$ ). However, the performance of the RR scheme with the choices (4.3) and (4.5) is only slightly better than RN with (4.3).

4.2. Optimization procedures. Our goal is to minimize the reduction factor $\rho$ in the different cases considered, by choosing the Robin parameters $\alpha_{f}, \alpha_{s}$ in the class of frequency dependent functions $\mathcal{A} \equiv\left\{\alpha(k)=\alpha_{M}+\alpha_{K} k^{2}, \alpha_{M}, \alpha_{K} \geq 0\right\}$. Functions in this class will lead to generalized Robin boundary conditions having a Mass and a Stiffness matrix on the boundary (see (4.1) and (4.2)).

In all the problems considered the reduction factor $\rho(k)$ can be split as the product of two factors: $\rho(k)=d_{1}(k) \cdot d_{2}(k)$ where

$$
\begin{array}{ll}
d_{1}(k)=\left|\frac{\frac{\rho_{s} H_{s}}{\Delta t}+\beta H_{s} \Delta t+G H_{s} \Delta t k^{2}-\alpha_{f}}{\frac{\rho_{s} H_{s}}{\Delta t}+\beta H_{s} \Delta t+G H_{s} \Delta t k^{2}+\alpha_{s}}\right| & \text { (Generalized string model) } \\
d_{1}(k)=\left|\frac{2 \alpha_{f} \delta-(\varepsilon+1) \Delta t}{2 \alpha_{s} \delta+(\varepsilon+1) \Delta t}\right| & \text { (Linear incompressible elasticity) }
\end{array}
$$

and

$$
\begin{array}{ll}
d_{2}(k)=\left|\frac{\rho_{f}-\alpha_{s} \Delta t k}{\rho_{f}+\alpha_{f} \Delta t k}\right|, & \text { (Potential flow) } \\
d_{2}(k)=\left|\frac{\alpha_{s} k-\mu \gamma_{f}\left(k+\gamma_{f}\right)}{\alpha_{f} k+\mu \gamma_{f}\left(k+\gamma_{f}\right)}\right|, & \text { (Stokes) }
\end{array}
$$

Since the global optimization of $\rho$ for all frequencies is too difficult, we try to minimize separately the two factors $d_{1}=d_{1}\left(k, \alpha_{f}, \alpha_{s}\right)$ and $d_{2}=d_{2}\left(k, \alpha_{f}, \alpha_{s}\right)$. The first can be associated to the structure problem and will lead to an optimal selection of $\alpha_{f} \in \mathcal{A}$ for all possible frequencies in $\left[0, k_{\max }\right]$ and all possible functions $\alpha_{s} \in \mathcal{A}$. Similarly, the second factor can be associated to the fluid problem and leads to the optimal selection of $\alpha_{s} \in \mathcal{A}$ uniformly with respect to $k$ and $\alpha_{f} \in \mathcal{A}$.

We set therefore the two optimization problems

$$
\begin{aligned}
& \alpha_{f}^{\text {opt }}(k)=\underset{\alpha_{f}(k) \in \mathcal{A}}{\operatorname{argmin}} \max _{k \in\left[0, k_{\max }\right]} \sup _{\alpha_{s} \in \mathcal{A}} d_{1}\left(k, \alpha_{f}(k), \alpha_{s}(k)\right), \\
& \alpha_{s}^{\text {opt }}(k)=\underset{\alpha_{s}(k) \in \mathcal{A}}{\operatorname{argmin}} \max _{k \in\left[0, k_{\max }\right]} \sup _{\alpha_{f} \in \mathcal{A}} d_{2}\left(k, \alpha_{f}(k), \alpha_{s}(k)\right) .
\end{aligned}
$$

Of course, by this procedure we will not get the global optimum but, hopfully, improve the heuristic choice of the Robin coefficients. 
4.2.1. Optimization of $\alpha_{f}$. We start with the optimization of the factor $d_{1}$.

Generalized string model. In this case we obtain $d_{1} \equiv 0$ for

$$
\alpha_{f}^{G S}=\frac{\rho_{s} H_{s}}{\Delta t}+\beta H_{s} \Delta t+G H_{s} \Delta t k^{2} .
$$

We point out that $\alpha_{f}^{G S}<\alpha_{f}^{\max }$, with $\alpha_{f}^{\max }$ given in (3.11). The previous expression represents the symbol, in the Fourier space, of the Dirichlet-to-Neumann operator in the structure domain. We notice that $\alpha_{f}^{G S}=\alpha_{f}^{\text {heur }}+G H_{s} \Delta t k^{2}$, and then the convergence analysis in Sect. 3.1 provides a correction of the optimal value of $\alpha_{f}$ with respect to the heuristic choice (4.3). The dependence of this correction on $k$ introduces in the Robin interface condition a differential term. In particular, we obtain condition (4.1) with

$$
\alpha_{f, M}^{G S}=\alpha_{f}^{h e u r}=\frac{\rho_{s} H_{s}}{\Delta t}+\beta H_{s} \Delta t, \quad \alpha_{f, K}^{G S}=G H_{s} \Delta t
$$

Therefore, the correction given by the convergence analysis of the potential flow/ generalized string problem requires to build a "stiffness" interface matrix.

Linear incompressible elasticity. We have $d_{1} \equiv 0$ for $\alpha_{f}(k)=\frac{(1+\varepsilon) \Delta t}{2 \delta}$, which, however, is not in the class $\mathcal{A}$. By considering an approximation of such expression for $H_{s}$ small, that is by using the expansions of $\varepsilon$ and $\delta$ in powers of $H_{s}$, given in (3.28), we obtain the optimized value

$$
\alpha_{f}^{L I E}=\Delta t \lambda \gamma_{s}^{2} H_{s}=\frac{\rho_{s} H_{s}}{\Delta t}+\beta H_{s} \Delta t+\lambda H_{s} \Delta t k^{2}
$$

which leads to $d_{1}=O\left(H_{s}^{3}\right)$. Observe that we obtain an expression very similar to $\alpha_{f}^{G S}$.

The previous result shows that the approximated reduction factor obtained by considering $H_{s}$ small is the same as the one that is obtained when a membrane model is considered for the structure.

4.2.2. Optimization of $\alpha_{s}$. We consider now the optimization of the factor $d_{2}$.

Potential flow. In this case the optimization problem reads

$\alpha_{s}^{\text {opt }}(k)=\underset{\alpha_{s}(k) \in \mathcal{A}}{\operatorname{argmin}} \max _{k \in\left[0, k_{\max }\right]} \sup _{\alpha_{f} \in \mathcal{A}}\left|\frac{\rho_{f}-\alpha_{s} \Delta t k}{\rho_{f}+\alpha_{f} \Delta t k}\right|=\underset{\alpha_{s}(k) \in \mathcal{A}}{\operatorname{argmin}} \max _{k \in\left[0, k_{\max }\right]}\left|1-\frac{\alpha_{s} \Delta t k}{\rho_{f}}\right|$

It is clear that for a generic choice $\alpha_{s}(k)=\alpha_{s, M}+\alpha_{s, K} k^{2}$ with $\alpha_{s, K}>0$ the factor $d_{2}(k)$ is unbounded for $k \rightarrow \infty$ (that is for $h \rightarrow 0$ ). Therefore we restrict the optimization problem only to frequency-independent functions, namely $\alpha_{s}(k)=\alpha_{s, M}$. In this case, we have

$$
\max _{k \in\left[0, k_{\text {max }}\right]}\left|1-\frac{\alpha_{s} \Delta t k}{\rho_{f}}\right|= \begin{cases}1 & \text { if } \alpha_{s, M} \leq \frac{2 \rho_{f}}{\Delta t k_{\text {max }}} \\ >1 & \text { otherwise }\end{cases}
$$

Therefore, no choice of $\alpha_{s, M}$ will lead to a factor $d_{2}$ strictly smaller than 1 , and we can chose any $\alpha_{s, M} \in\left[0, \frac{2 \rho_{f}}{\Delta t k_{\max }}\right]$ to have exactly $d_{2}=1$. However, in the expressions of $d_{1}, \alpha_{s}$ is at the denominator, so that in view of the optimization of the factor $d_{1}$ 
we have interest in choosing $\alpha_{s, M}$ as large as possible, leading to the optimized Robin coefficient

$$
\alpha_{s}^{P}=\frac{2 \rho_{f}}{\Delta t k_{\max }}
$$

Notice that this choice coincides with the upper bound of the stability interval given in (3.12)

Stokes flow. We proceed in a similar way as for the Potential flow. In this case we have

$$
\begin{aligned}
\alpha_{s}^{\text {opt }}(k)= & \underset{\alpha_{s}(k) \in \mathcal{A}}{\operatorname{argmin}} \max _{k \in\left[0, k_{\text {max }}\right]} \sup _{\alpha_{f} \in \mathcal{A}}\left|\frac{\alpha_{s} k-\mu \gamma_{f}\left(k+\gamma_{f}\right)}{\alpha_{f} k+\mu \gamma_{f}\left(k+\gamma_{f}\right)}\right|= \\
& =\underset{\alpha_{s}(k) \in \mathcal{A}}{\operatorname{argmin}} \max _{k \in\left[0, k_{\max }\right]}\left|\frac{\alpha_{s} k}{\mu \gamma_{f}\left(k+\gamma_{f}\right)}-1\right|
\end{aligned}
$$

and again we restrict the optimization only to constant values of $\alpha_{s}$ or otherwise the factor $d_{2}$ will be unbounded for $k \rightarrow \infty$.

Let us denote $f(k)=\frac{k}{\mu \gamma_{f}\left(k+\gamma_{f}\right)}=\frac{k}{\mu \sqrt{a+k^{2}}\left(k+\sqrt{a+k^{2}}\right)}$, with $a=\frac{\rho_{f}}{\mu \Delta t}$. The function $f$ has a global maximum in $[0, \infty)$ at $k^{*}=\sqrt{a \frac{\sqrt{5}-1}{2}}$. It is a straighforward calculation to show that

$$
\max _{k \in\left[0, k_{\text {max }}\right]}\left|\alpha_{s} f(k)-1\right|= \begin{cases}1 & \text { if } \alpha_{s} f\left(k^{*}\right) \leq 2 \\ >1 & \text { otherwise }\end{cases}
$$

Proceeding as for the potential flow we take the largest value for which $d_{2}=1$ leading to the optimized coefficient for the Stokes flow

$$
\alpha_{s}^{S}=\frac{2}{f\left(k^{*}\right)}=\frac{2}{\Delta t k^{*}} \sqrt{\rho_{f}+\mu \Delta t\left(k^{*}\right)^{2}}\left(\sqrt{\mu \Delta t} k^{*}+\sqrt{\rho_{f}+\mu \Delta t\left(k^{*}\right)^{2}}\right) .
$$

4.3. Alternative optimization procedure. Another possible way to proceed to derive optimized parameters for the RR algorithm consists in taking a different split of the reduction factor, namely $\rho\left(k, \alpha_{f}, \alpha_{s}\right)=\tilde{d}_{1}\left(k, \alpha_{f}\right) \cdot \tilde{d}_{2}\left(k, \alpha_{s}\right)$. In such a case, we can look for parameters $\alpha_{f}$ and $\alpha_{s}$ that optimize separately the two factors, namely:

$\alpha_{f}^{\text {opt }}(k)=\underset{\alpha_{f} \in \mathcal{A}}{\operatorname{argmin}} \max _{k \in\left[0, k_{\text {max }}\right]} \tilde{d}_{1}\left(k, \alpha_{f}(k)\right), \quad \alpha_{s}^{o p t}(k)=\underset{\alpha_{s} \in \mathcal{A}}{\operatorname{argmin}} \max _{k \in\left[0, k_{\max }\right]} \tilde{d}_{2}\left(k, \alpha_{s}(k)\right)$.

This optimization procedure will lead to the same values for $\alpha_{f}$ as in the previous section since these are the values that zero the factor $d_{1}$. However, this procedure will lead to different values for $\alpha_{s}$ which will depend, this time, both on the fluid and the structure problem chosen.

4.3.1. Optimization of $\alpha_{s}$. Potential flow / Generalized string. For the Potential Flow / Generalized string model, we have

$$
\tilde{d}_{2}\left(k, \alpha_{s}\right)=\left|\frac{\rho_{f}-\alpha_{s} \Delta t k}{\frac{\rho_{s} H_{s}}{\Delta t}+\beta H_{s} \Delta t+G H_{s} \Delta t k^{2}+\alpha_{s}}\right| .
$$


First, we observe that if $\alpha_{s}=\alpha_{s, M}+\alpha_{s, K} k^{2}$ with $\alpha_{s, K}>0$, the factor $\tilde{d}_{2}$ is unbounded for $k \rightarrow \infty$, so we restrict the optimization to constant values. Let us denote

$$
\tilde{f}\left(k, \alpha_{s}\right)=\frac{\rho_{f}-\alpha_{s} \Delta t k}{\frac{\rho_{s} H_{s}}{\Delta t}+\beta H_{s} \Delta t+G H_{s} \Delta t k^{2}+\alpha_{s}} .
$$

The function $\tilde{f}\left(k, \alpha_{s}\right)$ is such that $\tilde{f}\left(0, \alpha_{s}\right)=\frac{\rho_{f}}{\frac{\rho_{s} H_{s}}{\Delta t}+\beta H_{s} \Delta t+\alpha_{s}}>0, \frac{\partial \tilde{f}}{\partial k}\left(0, \alpha_{s}\right)<0$ and $\lim _{\kappa \rightarrow \infty} \tilde{f}\left(k, \alpha_{s}\right)=0$, and has exactly one global minimum in $k^{*}\left(\alpha_{s}\right) \in[0, \infty)$, with $\tilde{f}\left(k^{*}\left(\alpha_{s}\right), \alpha_{s}\right)<0$. We have, therefore that the optimal value $\alpha_{s}^{\text {opt }}$ satisfies (see Fig. 4.1)

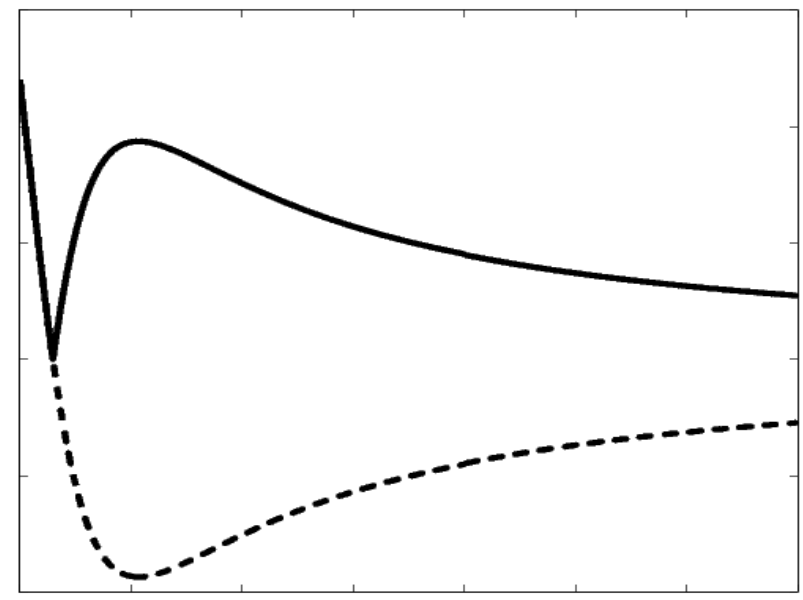

FIG. 4.1. $\tilde{f}$ as a function of $k$.

$$
\min _{\alpha_{s}} \max _{k}\left|\tilde{f}\left(k, \alpha_{s}\right)\right|=\min _{\alpha_{s}} \max \left\{\tilde{f}\left(0, \alpha_{s}\right),-f\left(k^{*}\left(\alpha_{s}\right), \alpha_{s}\right)\right\}
$$

We observe that $\tilde{f}\left(0, \alpha_{s}\right)$ is a decreasing function of $\alpha_{s}$ and $-\tilde{f}\left(k^{*}\left(\alpha_{s}\right), \alpha_{s}\right)$ is an increasing function of $\alpha_{s}$. Moreover, for $\alpha_{s}=0$ we have that $\tilde{f}(k, 0)>0$ is a monotone decreasing function in $k$, so that, thanks to the continuity of $\tilde{f}$, we have $\tilde{f}\left(0,0^{+}\right)>$ $-\tilde{f}\left(k^{*}\left(0^{+}\right), 0^{+}\right)$. Therefore, the minimum is achieved for

$$
\alpha_{s}^{P, 2} \text { such that } \tilde{f}\left(0, \alpha_{s}^{P, 2}\right)=-\tilde{f}\left(k^{*}\left(\alpha_{s}^{P, 2}\right), \alpha_{s}^{P, 2}\right) .
$$

This non-linear problem can be solved numerically given all the parameters of the fluid and structure model.

Stokes / Generalized String In this case we have

$$
\tilde{d}_{2}\left(k, \alpha_{s}\right)=\left|\frac{\mu \gamma_{f}\left(k+\gamma_{f}\right)-\alpha_{s} \Delta t k}{\frac{\rho_{s} H_{s}}{\Delta t}+\beta H_{s} \Delta t+G H_{s} \Delta t k^{2}+\alpha_{s}}\right| .
$$

Again, we observe that if $\alpha_{s}=\alpha_{s, M}+\alpha_{s, K} k^{2}$ with $\alpha_{s, K}>0$, the factor $\tilde{d}_{2}$ is unbounded for $k \rightarrow \infty$, so we restrict the optimization to constant values. Let us denote

$$
\tilde{f}\left(k, \alpha_{s}\right)=\frac{\mu \gamma_{f}\left(k+\gamma_{f}\right)-\alpha_{s} \Delta t k}{\frac{\rho_{s} H_{s}}{\Delta t}+\beta H_{s} \Delta t+G H_{s} \Delta t k^{2}+\alpha_{s}} .
$$


The function $\tilde{f}\left(k, \alpha_{s}\right)$ is such that $\tilde{f}\left(0, \alpha_{s}\right)=\frac{\rho_{f}}{\rho_{s} H_{s}+\beta H_{s} \Delta t^{2}+\alpha_{s} \Delta t}>0, \frac{\partial \tilde{f}}{\partial k}\left(0, \alpha_{s}\right)<0$ and $\lim _{k \rightarrow \infty} \tilde{f}\left(k, \alpha_{s}\right)=\tilde{F}:=\frac{\mu}{G H_{s} \Delta t}>0$, and has exactly one local minimum in $k^{*}\left(\alpha_{s}\right) \in[0, \infty)$, with $\tilde{f}\left(k^{*}\left(\alpha_{s}\right), \alpha_{s}\right)<0$. Let us assume that $k_{\max }$ is big enough to approximate $\tilde{f}\left(k_{\max }, \alpha_{s}\right)$ with $\tilde{F}$. We have, therefore that the optimal value $\alpha_{s}^{\text {opt }}$ satisfies

$$
\min _{\alpha_{s}} \max _{k}\left|\tilde{f}\left(k, \alpha_{s}\right)\right|=\min _{\alpha_{s}} \max \left\{\tilde{f}\left(0, \alpha_{s}\right),-f\left(k^{*}\left(\alpha_{s}\right), \alpha_{s}\right), \tilde{F}\right\} .
$$

Again, $\tilde{f}\left(0, \alpha_{s}\right)$ is a decreasing function of $\alpha_{s}$ and $-\tilde{f}\left(k^{*}\left(\alpha_{s}\right), \alpha_{s}\right)$ is an increasing function of $\alpha_{s}$, with $\tilde{f}\left(0,0^{+}\right)>-\tilde{f}\left(k^{*}\left(0^{+}\right), 0^{+}\right)$. Moreover, under the hypothesis on the data $4 \mu^{2} \rho_{s} \beta>\rho_{f}^{2} G^{2}$, we have that $\tilde{f}(0,0)>\tilde{F}$. Therefore, under this hypothesis, minimum is achieved for

$$
\alpha_{s}^{o p t}=\left\{\begin{array}{lll}
\alpha_{s, 1} & \text { if } & \tilde{f}\left(0, \alpha_{s, 1}\right)>\tilde{F}, \\
\alpha_{s, 2} & \text { if } & \tilde{F}>\tilde{f}\left(0, \alpha_{s, 1}\right),
\end{array}\right.
$$

where

$$
\alpha_{s, 1} \text { such that } \tilde{f}\left(0, \alpha_{s, 1}\right)=-\tilde{f}\left(k^{*}\left(\alpha_{s, 1}\right), \alpha_{s, 1}\right)
$$

and

$$
\alpha_{s, 2} \text { such that } \tilde{f}\left(0, \alpha_{s, 2}\right)=\tilde{F} \text {. }
$$

Again, this non-linear problem can be solved numerically given all the parameters of the fluid and structure model.

5. Numerical Results. In this section we present some numerical results with the aim of testing the optimized parameters found in the previous analysis. We focus on two-dimensional numerical simulations, even if the application of the proposed parameters to three-dimensional problems is under investigation. This is in agreement with the fact that the convergence analysis has been performed in two-dimensional domains. However, the application of the optimal coefficients found in this analysis to three-dimensional cases is under investigation.

For the structure, we consider the following linear elasticity equation

$$
\rho_{s} \partial_{t t} \boldsymbol{\eta}-\lambda_{1} \nabla \cdot\left(\nabla \boldsymbol{\eta}+(\nabla \boldsymbol{\eta})^{t}\right)-\lambda_{2} \nabla \cdot((\nabla \cdot \boldsymbol{\eta}) I)+\beta \boldsymbol{\eta}=\mathbf{0},
$$

where the Lamé constants are given by (2.1) and a reaction term $\beta$, arising in the derivation of the generalized string model in the $3 \mathrm{D}$ case, is introduced also in $2 \mathrm{D}$ to reproduce the behaviour of a cylindrical structure. For its expression we use the particolarization of (4.4) to the rectangular domain, that is $\beta=\frac{E}{\left(1-\nu^{2}\right) R^{2}}$, where $R$ is half the heigth of the rectangle.

For the numerical solution we use a 2D Finite Element Code written in Matlab at MOX - Dipartimento di Matematica - Politecnico di Milano and at the Department of Information Technology and Mathematical Methods - Università degli Studi di Bergamo. Moreover, we consider $\mathbb{P}_{1}-$ bubble $/ \mathbb{P}_{1}$ elements for the fluid and $\mathbb{P}_{1}$ elements for the structure. We use the residual normalized to the initial one as stopping criterion (see [3]), with a tolerance equal to $10^{-4}$.

In all the cases, we prescribe an impulse pressure jump between the inlet and the outlets

$$
\Delta P(t)= \begin{cases}10^{4} \text { dyne } / \mathrm{cm}^{2} & t \leq 0.005 \mathrm{~s} \\ 0 & t>0.005 \mathrm{~s}\end{cases}
$$


Finally, we set $\mu=0.035$ poise, $\rho_{f}=1 \mathrm{~g} / \mathrm{cm}^{3}$ and the following set of reference values: $\Delta t=10^{-3} \mathrm{~s}, \rho_{s}=1.1 \mathrm{~g} / \mathrm{cm}^{3}, E=1.3 \cdot 10^{6}$ dyne $/ \mathrm{cm}^{2}$ and the thickness of the structure $H_{s}=0.1 \mathrm{~cm}$.

5.1. Results in a rectangle domain. The numerical simulations of this section are performed in a rectangular domain both for the fluid and for the two structures, whose size is $6 \times 1 \mathrm{~cm}$ and $6 \times H_{s} \mathrm{~cm}$, respectively (see Fig. 5.1). We set $\nu=0.3, R=$

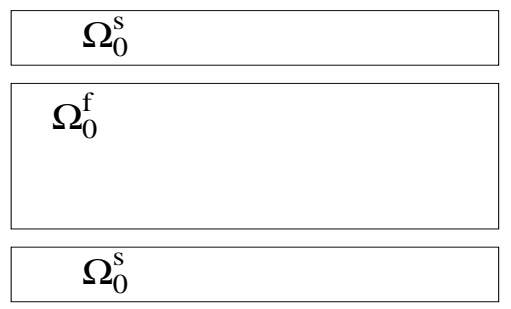

FIG. 5.1. Computational fluid and structure domains.

0.5 and the space discretization parameter is $h=H_{s} / 2$, both for the fluid and the structure domains.

The aim of this test is to compare the performance of the proposed parameters given in (4.6), (4.7) and (4.8) with respect to the heuristic choice. In particular, for the reference values, we have the following values: $\alpha_{f, M}^{G S}=681.43, \alpha_{f, K}^{G S}=41.12, \alpha_{s}^{P}=$ 31.83 and $\alpha_{s}^{S}=38.95$.

In Table 5.1 we show the average number of iterations to reach convergence having considered as final time $T=0.008 \mathrm{~s}$. In brackets, we show the computational time, normalized to that obtained with the heuristic choice of $\alpha_{f}$ and with $\alpha_{s}=0$.

\begin{tabular}{|c|cccc|}
$\alpha_{f}=$ & $\alpha_{f}^{\text {heur }}$ & $\alpha_{f}^{G S}$ & $\alpha_{f}^{G S}$ & $\alpha_{f}^{G S}$ \\
$\alpha_{s}=$ & 0 & 0 & $\alpha_{s}^{P}$ & $\alpha_{s}^{S}$ \\
\hline Ref. & $5.87(1.00)$ & $5.37(0.91)$ & $4.62(0.79)$ & $4.50(0.77)$ \\
$\rho_{s}=0.1$ & $6.00(1.00)$ & $5.62(0.94)$ & $4.87(0.81)$ & $4.87(0.81)$ \\
$\rho_{s}=0.01$ & $6.00(1.00)$ & $5.75(0.96)$ & $5.00(0.83)$ & $4.87(0.81)$ \\
$H_{s}=0.05$ & $6.75(1.00)$ & $5.87(0.87)$ & $5.00(0.74)$ & $5.00(0.74)$ \\
$H_{s}=0.0125$ & $10.75(1.00)$ & $7.87(0.73)$ & $7.62(0.71)$ & $5.75(0.53)$ \\
$\Delta t=5 \cdot 10^{-4}$ & $7.00(1.00)$ & $5.69(0.81)$ & $4.94(0.71)$ & $4.87(0.70)$ \\
$\Delta t=2.5 \cdot 10^{-4}$ & $6.94(1.00)$ & $5.56(0.80)$ & $4.81(0.69)$ & $4.41(0.64)$ \\
$E=6.5 \cdot 10^{5}$ & $6.50(1.00)$ & $5.62(0.86)$ & $4.87(0.75)$ & $4.87(0.75)$ \\
$E=1.3 \cdot 10^{5}$ & $7.50(1.00)$ & $5.50(0.73)$ & $4.37(0.58)$ & $5.00(0.67)$
\end{tabular}

Average number of iterations and relative CPU times (in brackets) for the rectangular domain for different values of some parameters. Ref. means reference values.

These results show that the Robin-Robin schemes are very robust with respect to the added-mass effect. Indeed also for structure density 100 times lighter than the fluid one, convergence is reached without any relaxation and with a small number of iterations. However, a worsening in the performance is observed when the structure thickness $H_{s}$, the time step $\Delta t$ and the Young modulus $E$ decrease. Moreover, these results show that there is a systematic improvement in the performance of the RobinNeumann scheme when adopting the optimized value $\alpha_{f}^{G S}$ instead of the heuristic 
one, with a saving in the computational time up to $27 \%$. A further improvement is obtained by considering the Robin-Robin scheme with coefficient $\alpha_{s}^{P}$ or $\alpha_{s}^{S}$. In these cases the saving in computational time is up to $47 \%$. We point out that the proposed choice is robust, since numerical simulation highlightes that the number of iterations is independent of the value of the space discretization parameter $h$.

In the second set of simulations, we want to compare the two different optimization strategies proposed for the parameter $\alpha_{s}$ in Sect. 4.2 and 4.3, respectively. We limit the analysis to the Potential flow case, that is we compare the performances of the RR scheme with $\alpha_{s}$ given by (4.7) and (4.9).

In Table 5.2 we show the average number of iterations to reach convergence having considered 16 time steps, by changing the Young modulus and the time step. These

\begin{tabular}{|c|cc|}
$\alpha_{s}=$ & $\alpha_{s}^{P}$ & $\alpha_{s}^{P, 2}$ \\
\hline Ref. & 4.62 & 10.87 \\
$H_{s}=0.0125$ & 7.73 & $\mathrm{X}$ \\
$\Delta t=6.4 \cdot 10^{-5}$ & 6.00 & 5.00 \\
$\Delta t=3.2 \cdot 10^{-5}$ & 10.94 & 9.00 \\
$E=1.3 \cdot 10^{4}$ & 4.62 & 6.37 \\
$E=1.3 \cdot 10^{3}$ & 26.75 & 15.00
\end{tabular}

Average number of iterations to perform 16 time steps for the rectangular domain. Ref. means reference values. $X$ means no convergence.

results clearly show that the optimized parameter $\alpha_{s}^{P, 2}$ shows a better performance when the structure stiffness term is small in comparison to the other terms. However, as highlighted by the performances obtained with the reference values and by the non-convergence with $H_{s}=0.0125$, this choice seems less robust than $\alpha_{s}^{P}$.

5.2. An application to a $2 \mathrm{D}$ bifurcation geometry. In this section we investigate the performance of Robin-Robin algorithm with optimized parameters considering a $2 \mathrm{D}$ geometry which is an idealization of a realistic domain, namely the human carotid. We use the reference values described in the previous sections and we set $\nu=0.49$ in order to model the quasi-incompressibility of arterial vessels.

In Figure 5.2 the pressure in the deformed fluid domain is shown, at three time instants. In Table 5.3 we show the average number of iterations and the normalized
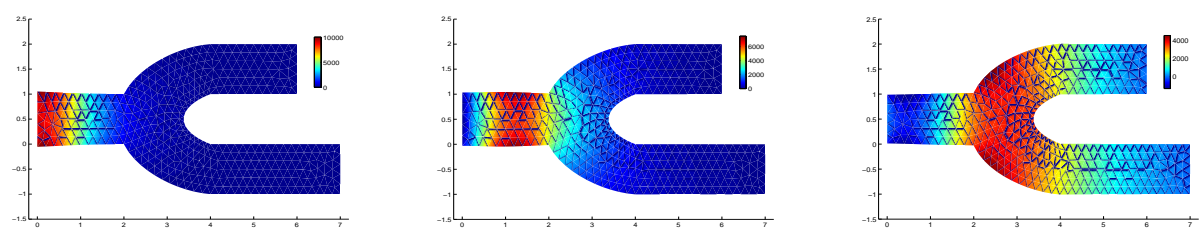

FIG. 5.2. Pressure in the deformed fluid domain obtained with $\alpha_{f}=\alpha_{f}^{G S}$ and $\alpha_{s}=\alpha_{s}^{P}$; $t=0.004$ (left), $t=0.008 s$ (middle), $t=0.012 \mathrm{~s}$ (right).

computational times (in brackets) for two values of the structure thickness, having considered as final time $T=0.012 s$.

These results show that the improvement in the performances of RR scheme in using the optimazed parameters $\alpha_{f}^{G S}, \alpha_{s}^{P}$ and $\alpha_{s}^{S}$ is even slightly higher than for the rectangular case. 


\begin{tabular}{|c|cccc|}
$\alpha_{f}=$ & $\alpha_{f}^{\text {heur }}$ & $\alpha_{f}^{G S}$ & $\alpha_{f}^{G S}$ & $\alpha_{f}^{G S}$ \\
$\alpha_{s}=$ & 0 & 0 & $\alpha_{s}^{P}$ & $\alpha_{s}^{S}$ \\
\hline Ref. & $13.08(1.00)$ & $9.92(0.76)$ & $7.00(0.54)$ & $6.75(0.52)$ \\
$H_{s}=0.05$ & $23.25(1.00)$ & $14.08(0.61)$ & $13.50(0.58)$ & $12.67(0.54)$
\end{tabular}

TABLE 5.3

Average number of iterations and relative CPU times (in brackets) for the carotid domain for different values of the structure thickness $H_{s}$. Ref. means reference values.

6. Conclusions. In this work, we have proposed new convergence analysis of the Robin-Robin partitioned procedure for the Fluid-Structure Interaction problem. In particular, we consider 2D generalized Stokes problem both for the fluid and for structure. These analysis improve and generalize the ones presented in $[3,6]$ which referred to a potential flow model for the fluid and a 1D reduced model for the structure. The analysis have been performed applying the Fourier transform in one direction, and highlight the dependence of the Dirichlet-Neumann scheme on the ratio between the densities of the fluid and of the structure, and the robustness of a general $\mathrm{RR}$ scheme with respect to this ratio.

Moreover, in the spirit of the Optimized Schwarz Methods, the reduction factor of the analysis have been minimized by determining optimal values of the parameters in the Robin transmission conditions.

The $2 \mathrm{D}$ numerical results show that the proposed optimized parameters improve considerably the speed of convergence of RR schemes.

\section{REFERENCES}

[1] A. Alonso-Rodriguez and L. Gerardo-Giorda. New non-overlapping domain decomposition methods for the time-harmonic Maxwell system. SIAM Journal on Scientific Computing, 28(1):102-122, 2006.

[2] S. Badia, F. Nobile, and C. Vergara. Robin-Robin preconditioned Krylov methods for fluidstructure interaction problems. Computer Methods in Applied Mechanics and Engineering, 198(33-36):2768-2784, 2009.

[3] S. Badia, F. Nobile, and Ch. Vergara. Fluid-structure partitioned procedures based on Robin transmission conditions. Journal of Computational Physics, 227:7027-7051, 2008.

[4] S. Badia, A. Quaini, and A. Quarteroni. Modular vs. non-modular preconditioners for fluidstructure systems with large added-mass effect. Computer Methods in Applied Mechanics and Engineering, 197:4216-4232, 2008

[5] S. Badia, A. Quaini, and A. Quarteroni. Splitting methods based on algebraic factorization for fluid-structure interaction. SIAM Journal on Scientific Computing, 30(4):1778-1805, 2008.

[6] P. Causin, J.F. Gerbeau, and F. Nobile. Added-mass effect in the design of partitioned algorithms for fluid-structure problems. Computer Methods in Applied Mechanics and Engineering, 194(42-44):4506-4527, 2005.

[7] Q.P. Deng. An analysis for a nonoverlapping domain decomposition iterative procedure. SIAM Journal on Scientific Computing, 18:1517-1525, 1997.

[8] S. Deparis, M. Discacciati, G. Fourestey, and A. Quarteroni. Fluid-structure algorithms based on Steklov-Poincaré operators. Computer Methods in Applied Mechanics and Engineering, 195(41-43):5797-5812, 2006.

[9] J. Donea. An arbitrary Lagrangian-Eulerian finite element method for transient dynamic fluidstructure interaction. Computer Methods in Applied Mechanics and Engineering, 33:689723, 1982.

[10] M.A. Fernández, J.F. Gerbeau, and C. Grandmont. A projection semi-implicit scheme for the coupling of an elastic structure with an incompressible fluid. International Journal for Numerical Methods in Engineering, 69(4):794-821, 2007.

[11] M.A. Fernández and M. Moubachir. A Newton method using exact Jacobians for solving fluidstructure coupling. Computers \& Structures, 83(2-3):127-142, 2005. 
[12] C. Forster, W. Wall, and E. Ramm. Artificial added mass instabilities in sequential staggered coupling of nonlinear structures and incompressible viscous flow. Computer Methods in Applied Mechanics and Engineering, 196(7):1278-1293, 2007.

[13] M.J. Gander. Optimized Schwarz methods. SIAM Journal on Numerical Analysis, 44(2):699731, 2006.

[14] M.J. Gander, F. Magoulès, and F.Nataf. Optimized Schwarz methods without overlap for the Helmholtz equation. SIAM Journal on Scientific Computing, 24:38-60, 2002.

[15] L. Gerardo Giorda, P. Le Tallec, and F.Nataf. A Robin-Robin preconditioner for advectiondiffusion equations with discontinuous coefficients. Computer Methods in Applied Mechanics and Engineering, 193,(9-11):745-764, 2004.

[16] W. Guo and L.S. Hou. Generalizations and accelerations of Lions' nonoverlapping domain decomposition method for linear elliptic PDEs. SIAM Journal on Numerical Analysis, 41(6):2056-2080, 2003.

[17] T. J. R. Hughes, W. K. Liu, and T. K. Zimmermann. Lagrangian-Eulerian finite element formulation for incompressible viscous flows. Computer Methods in Applied Mechanics and Engineering, 29(3):329-349, 1981.

[18] P.L. Lions. On the Schwartz alternating method iii. In T. Chan, R. Glowinki, J. Periaux, and O.B. Widlund, editors, Proceedings of the Third International Symposium on Domain Decomposition Methods for PDE's, pages 202-223. Siam, Philadelphia, 1990.

[19] F. Nobile. Numerical approximation of fluid-structure interaction problems with application to haemodynamics. PhD thesis, École Polytechnique Fédérale de Lausanne, 2001. Thesis ${ }^{\circ}$ 2458.

[20] F. Nobile and C. Vergara. An effective fluid-structure interaction formulation for vascular dynamics by generalized Robin conditions. SIAM Journal on Scientific Computing, 30(2):731-763, 2008.

[21] S. Piperno and C. Farhat. Partitioned prodecures for the transient solution of coupled aeroelastic problems-Part II: energy transfer analysis and three-dimensional applications. Computer Methods in Applied Mechanics and Engineering, 190:3147-3170, 2001.

[22] A. Quarteroni and L. Formaggia. Mathematical Modelling and Numerical Simulation of the Cardiovascular System, volume Modelling of Living Systems, Handbook of Numerical Analysis. Elsevier Science, 2003.

[23] P. Le Tallec and J. Mouro. Fluid structure interaction with large structural displacements. Computer Methods in Applied Mechanics and Engineering, 190:3039-3067, 2001. 